TRANSACTIONS OF THE

AMERICAN MATHEMATICAL SOCIETY

Volume 354, Number 5, Pages 2055-2077

S 0002-9947(01)02925-7

Article electronically published on December 27, 2001

\title{
THE SUPER ORDER DUAL OF AN ORDERED VECTOR SPACE AND THE RIESZ-KANTOROVICH FORMULA
}

\author{
CHARALAMBOS D. ALIPRANTIS AND RABEE TOURKY
}

\begin{abstract}
A classical theorem of F. Riesz and L. V. Kantorovich asserts that if $L$ is a vector lattice and $f$ and $g$ are order bounded linear functionals on $L$, then their supremum (least upper bound) $f \vee g$ exists in $L^{\sim}$ and for each $x \in L_{+}$it satisfies the so-called Riesz-Kantorovich formula:

$$
[f \vee g](x)=\sup \left\{f(y)+g(z): y, z \in L_{+} \text {and } y+z=x\right\} \text {. }
$$

Related to the Riesz-Kantorovich formula is the following long-standing problem: If the supremum of two order bounded linear functionals $f$ and $g$ on an ordered vector space exists, does it then satisfy the Riesz-Kantorovich formula?

In this paper, we introduce an extension of the order dual of an ordered vector space and provide some answers to this long-standing problem. The ideas regarding the Riesz-Kantorovich formula owe their origins to the study of the fundamental theorems of welfare economics and the existence of competitive equilibrium. The techniques introduced here show that the existence of decentralizing prices for efficient allocations is closely related to the abovementioned problem and to the properties of the Riesz-Kantorovich formula.
\end{abstract}

\section{INTRODUCTION}

Consider two ordered (real) vector spaces $L$ and $M$, and let $T: L \rightarrow M$ be a linear operator. The operator $T$ is said to be positive, in symbols $T \geq 0$, if $T x \geq 0$ holds for each $x \geq 0$. Also, $T$ is a regular operator if $T$ can be written as a difference of two positive operators. Similarly, $T$ is called order bounded if it carries order bounded subsets of $L$ to order bounded subsets of $M$. If $\mathcal{L}^{\sim}(L, M)$ and $\mathcal{L}^{r}(L, M)$ denote the vector spaces of all order bounded and regular operators from $L$ to $M$, respectively, then we have $\mathcal{L}^{r}(L, M) \subseteq \mathcal{L}^{\sim}(L, M)$. Moreover, $\mathcal{L}^{r}(L, M)$ and $\mathcal{L}^{\sim}(L, M)$ are both ordered vector spaces when equipped with the ordering $\geq$ defined by $S \geq T$ whenever $S-T \geq 0$. (The inequality $S \geq T$ is also expressed by saying that $S$ dominates $T$.) The ordered vector space $L^{\sim}=\mathcal{L}^{\sim}(L, \mathbb{R})$ is called the order dual of $L$. The vector space of all regular linear functionals on $L$ is denoted $L^{r}=\mathcal{L}^{r}(L, \mathbb{R})$.

Received by the editors April 20, 2000 and, in revised form, August 16, 2001.

2000 Mathematics Subject Classification. Primary 46A40, 46E99, 47B60; Secondary 91B50.

Key words and phrases. Ordered vector space, super order dual, Riesz-Kantorovich formula, decentralizing prices.

The research of C. D. Aliprantis is supported by NSF Grant EIA-007506, and the research of R. Tourky is funded by Australian Research Council Grant A00103450. 
As mentioned in the abstract, this work is associated with the following famous theorem due to F. Riesz [28] and L. V. Kantorovich [20]; for a proof see [11] Theorem 1.13, p. 12]. (Recall that the Riesz Decomposition Property asserts that: If $0 \leq y \leq x_{1}+x_{2}$ and $x_{1}, x_{2} \in L_{+}$, then there exist $y_{1}, y_{2} \in L_{+}$satisfying $y=y_{1}+y_{2}$, $0 \leq y_{1} \leq x_{1}$, and $0 \leq y_{2} \leq x_{2}$.)

Theorem 1.1 (Riesz-Kantorovich). If $L$ is an ordered vector space with the Riesz Decomposition Property and $M$ is a Dedekind complete vector lattice, then the ordered vector space $\mathcal{L}^{\sim}(L, M)$ is a Dedekind complete vector lattice. Moreover, if $S, T \in \mathcal{L}^{\sim}(L, M)$, then for each $x \in L_{+}$we have

$$
\begin{aligned}
& {[S \vee T](x)=\sup \{S y+T(x-y): 0 \leq y \leq x\},} \\
& {[S \wedge T](x)=\inf \{S y+T(x-y): 0 \leq y \leq x\} .}
\end{aligned}
$$

The above formulas describing the lattice operations in $\mathcal{L}^{\sim}(L, M)$ are known as the Riesz-Kantorovich formulas. Related to these formulas is the following longstanding unsolved question.

- If $L$ and $M$ are vector lattices with $M$ not Dedekind complete and the supremum (least upper bound) $S \vee T$ of two operators $S, T \in \mathcal{L}^{\sim}(L, M)$ exists in $\mathcal{L}^{\sim}(L, M)$, does it then satisfy the Riesz-Kantorovich formula?

This question and some related ones have been studied extensively in the past by many investigators; see for instance [1, 2, 3, 4, 15, 6, 30. Although some progress has been made and some partial answers have been given over the last fifty years, a satisfactory solution to this problem has not yet been obtained.

This paper provides some answers to this question when $L$ is an ordered topological vector space and $M=\mathbb{R}$. Notably, many of the ideas presented here regarding the Riesz-Kantorovich formulas owe their origins to recent advances in the theory of general economic equilibrium. We shall illustrate briefly below the connection between economic theory and the Riesz-Kantorovich formula. 1

For the rest of the paper, $L$ will denote an ordered vector space with a generating positive cone $L_{+}$, i.e., $L=L_{+}-L_{+}$. Let $f$ and $g$ be a pair of order bounded linear functionals on $L$ (i.e., $f, g \in L^{\sim}$ ) for which the supremum $f \vee g$ exists in $L^{\sim}$. We shall say that $f \vee g$ satisfies the Riesz-Kantorovich formula if for any $\omega \in L_{+}$we have

$$
[f \vee g](\omega)=\sup _{0 \leq x \leq \omega}[f(x)+g(\omega-x)] .
$$

In this case, the long-standing open problem regarding the Riesz-Kantorovich formula can be restated as follows:

- Are there an ordered vector space $L$ and two order bounded linear functionals $f$ and $g$ for which the supremum $f \vee g$ exists in $L^{\sim}$ and fails to satisfy the Riesz-Kantorovich formula?

To answer this question, we introduce a new collection of real-valued functions defined on the positive cone $L_{+}$that includes all order bounded linear functionals on $L$. This collection (which we shall denote $\mathcal{H}_{s}^{\sim}$ and call the super order dual of $L)$ consists of all order upper bounded, positively homogeneous, and super-additive functions from $L_{+}$to $\mathbb{R}$. The super order dual $\mathcal{H}_{s}^{\sim}$ inherits the partial ordering

${ }^{1}$ L. V. Kantorovich and T. C. Koopmans shared the 1975 Nobel Prize in Economics "for their contributions to the theory of optimum allocation of resources." 
of $\mathbb{R}^{L_{+}}$. That is, if $\psi, \phi \in \mathcal{H}_{s}^{\sim}$, then $\psi$ dominates $\phi$ if $\psi(x) \geq \phi(x)$ holds for all $x \in L_{+}$. This partial ordering has the following basic property.

Proposition 1. The collection of all positive functions in $\mathcal{H}_{s}^{\sim}$ is a lattice, and the supremum of every pair of positive functions satisfies the Riesz-Kantorovich formula, i.e., for $0 \leq \psi, \phi \in \mathcal{H}_{s}^{\sim}$ and each $\omega \in L_{+}$we have

$$
[\psi \vee \phi](\omega)=\sup _{0 \leq x \leq \omega}[\psi(x)+\phi(\omega-x)]
$$

If we also assume that $L$ is equipped with a Hausdorff locally convex topology $\tau$ for which the order intervals are $\tau$-bounded and the positive cone $L_{+}$is closed, then we can distinguish one more collection of functions from $L_{+}$to $\mathbb{R}$. This is the collection $\mathcal{H}_{s}^{\prime}$ of all upper $\tau$-semicontinuous, positively homogeneous, and superadditive functions from $L_{+}$to $\mathbb{R}$. We call this collection the super topological dual of $L$. Clearly, $L^{\prime} \subseteq \mathcal{H}_{s}^{\prime}$, where $L^{\prime}=(L, \tau)^{\prime}$ is the topological dual of $(L, \tau)$. Also, $\mathcal{H}_{s}^{\prime} \subseteq \mathcal{H}_{s}^{\sim}$. We shall establish the following result on the embedding of $L^{\prime}$ into $\mathcal{H}_{s}^{\prime}$.

Proposition 2. The canonical embedding of $L^{\prime}$ into $\mathcal{H}_{s}^{\prime}$ preserves finite supremum, i.e., if $f$ and $g$ are continuous linear functionals on $L$ and the supremum $f \vee g$ exists in $L^{\prime}$, then

$$
\left.(f \vee g)\right|_{L_{+}}=\left.\left.f\right|_{L_{+}} \vee g\right|_{L_{+}},
$$

where the supremum on the right is taken in $\mathcal{H}_{s}^{\prime}$.

Recently, in the study of market equilibrium there has been some attention on weakening the pervasive requirement that commodity spaces be vector lattices 2 Indeed, as shown in [14], one can drop all vector lattice theoretic considerations if linear prices are replaced by the possibly non-linear Riesz-Kantorovich functionals. For any $m$-tuple of functions $f=\left(f_{1}, \ldots, f_{m}\right)$ in $\mathcal{H}_{s}^{\sim}$ we define its RieszKantorovich functional $\mathcal{R}_{f}: L_{+} \rightarrow \mathbb{R}$ via the formula

$$
\mathcal{R}_{f}(\omega)=\sup \left\{f_{1}\left(x_{1}\right)+\cdots+f_{m}\left(x_{m}\right): x_{i} \in L_{+} \text {for each } i \text { and } \sum_{i=1}^{m} x_{i} \leq \omega\right\} .
$$

By using the Riesz-Kantorovich functionals as the generalized measures of economic value (i.e., as prices) one can extend the fundamental results on the Arrow-DebreuMcKenzie model of general competitive equilibrium beyond vector lattices and to general ordered vector spaces 3 Notice that the Riesz-Kantorovich functional is always an element of $\mathcal{H}_{s}^{\sim}$; in fact, it is the supremum of the set $\left\{0, f_{1}, \ldots, f_{m}\right\}$ in $\mathcal{H}_{s}^{\sim}$. This paper also establishes the following result, which connects Proposition 1 on the lattice structure of $\mathcal{H}_{s}^{\sim}$ and the embedding result for $\mathcal{H}_{s}^{\prime}$ in Proposition 2 .

\footnotetext{
${ }^{2}$ Infinite dimensional equilibrium analysis in economics is typically done in a vector lattice setting. This is in sharp contrast to the coordinate-free finite dimensional analysis of G. Debreu [17]. In fact, all the important results in the literature that establish the fundamental theorems of welfare economics or the existence of market equilibrium utilize in a non-trivial way the lattice structure of the commodity space and its dual. For example, Mas-Colell in [24] uses the local solidness and the Riesz Decomposition Property of the Riesz commodity space; for more on these techniques see [8, 9].

${ }^{3}$ In [14] it is also proposed to interpret the Riesz-Kantorovich functional as the revenue function from a discriminatory price auction, which is a generalization of the U.S. Treasury Bill Auction. See also 13$]$.
} 
Proposition 3. Let $f=\left(f_{1}, f_{2}\right)$ be a pair of positive continuous linear functionals on $L$. If the supremum $f_{1} \vee f_{2}$ exists in $L^{\prime}$, then it satisfies the RieszKantorovich formula if and only if the Riesz-Kantorovich functional $\mathcal{R}_{f}$ is upper $\tau$-semicontinuous.

In particular, if the order intervals of $L$ are weakly compact and either

a. $L_{+}$has an interior point, or

b. $L$ is an ordered Fréchet space,

then $f_{1} \vee f_{2}$ must satisfy the Riesz-Kantorovich formula. Moreover, if order intervals of $L$ are not weakly compact, then $f_{1} \vee f_{2}$ must satisfy the Riesz-Kantorovich formula in the algebraic dual $\left(L^{\prime}\right)^{*}$ of $L^{\prime}$.

As we shall see, continuity conditions for the Riesz-Kantorovich functionals offer a rich insight into the structure of partially ordered vector spaces that are not vector lattices. For example, it turns out that the order dual of $L$ coincides with the regular dual of $L$ (as in the case of vector lattices) if and only if every Riesz-Kantorovich functional is upper semicontinuous. Furthermore, if the RieszKantorovich functionals are upper semicontinuous, then the order intervals in $L$ are $\sigma\left(\left(L^{\prime}\right)^{*}, L^{\prime}\right)$-dense in the order intervals of $\left(L^{\prime}\right)^{*}$; this extends an important property of locally convex-solid vector lattices.

It has become apparent that there is a remarkable relationship between the fundamental theorems of welfare economics and the properties of the Riesz-Kantorovich functional. These fundamental theorems state that an allocation of resources is efficient if and only if the allocation can be decentralized by a price system, i.e., by a continuous linear functional. The welfare theorems are the basic results that provide conceptual foundations for our understanding of efficiency and decentralization of resources. Moreover, all applied economic policy analysis relies implicitly on the foundations provided by these theorems. For details about the welfare theorems, see [9].

This paper also establishes the following result, which has many consequences in economic theory.

Proposition 4. If $L$ is an ordered Fréchet space with weakly compact intervals, then for any $m$-tuple $f=\left(f_{1}, \ldots, f_{m}\right)$ of linear functionals in $L^{\sim}$ and any $\omega \in L_{+}$ we have

$$
\inf _{g \geq f_{1}, \ldots, g \geq f_{m}} g(\omega)=\mathcal{R}_{f}(\omega) .
$$

Surprisingly enough, it can be shown (see 12] that the so-called third fundamental theorem of welfare economics is basically equivalent to the statement that for any $m$-tuple of linear functionals $f=\left(f_{1}, \ldots, f_{m}\right)$ in $L^{\sim}$ and any $\omega \in L_{+}$a solution to the minimization problem in $(\star)$ exists, i.e., there exists some $g^{*} \in L^{\sim}$ satisfying $g^{*} \geq f_{i}$ for each $i$ and $g^{*}(\omega)=\mathcal{R}_{f}(\omega)$.

The material in this paper is organized as follows. The mathematical preliminaries are included in Section 2, Section 3 introduces the super order dual of an ordered vector space, which is an extension of the order dual. The lattice properties of the super order dual are discussed in Section 4 Our main embedding result and its consequences can be found in Sections 5, 6] and 7 Examples and final remarks are in the concluding section of this paper. 


\section{Mathematical Preliminaries}

For notation and terminology not explained below regarding ordered vector spaces we refer the reader to the monographs [19, 23, [25, 31]. In this work the letter $L$ will denote exclusively an ordered vector space. That is, $L$ is a real vector space equipped with an order relation $\geq$ such that $x \geq y$ implies $x+z \geq y+z$ for all $z \in L$ and $\alpha x \geq \alpha y$ for each $\alpha \geq 0$. The convex set $L_{+}=\{x \in L: x \geq 0\}$ is called the positive cone of $L$ and its members are referred to as positive vectors 4 The positive cone $L_{+}$satisfies the following properties:

1. $L_{+}+L_{+} \subseteq L_{+}$,

2. $\alpha L_{+} \subseteq L_{+}$for each $\alpha \geq 0$, and

3. $L_{+} \cap\left(-L_{+}\right)=\{0\}$.

Any subset $C$ of a vector space $X$ that satisfies the above properties (1), (2), and (3) is called a convex cone. Every convex cone $C$ induces a natural order $\geq$ on $X$ by letting $x \geq y$ whenever $x-y \in C$. This order makes $X$ an ordered vector space satisfying $X_{+}=C$. In other words, an ordered vector space is completely characterized by its positive cone. Any subset $C$ of a vector space that satisfies the above properties (1) and (2) is called a wedge.

The cone $L_{+}$is said to be generating if for each $x \in L$ there exist $y, z \in L_{+}$such that $x=y-z$. Equivalently, $L_{+}$is generating if every vector of $L$ is dominated by some positive vector. In this work we shall assume that:

- The cone $L_{+}$is always generating.

A (nonempty) subset $A$ of $L$ is bounded from above (resp. from below) if there exists some $x \in L$ satisfying $a \leq x$ (resp. $x \leq a$ ) for all $a \in A$; the vector $x$ is called an upper bound (resp. lower bound) of $A$. A set is order bounded if it is bounded from above and below. Sets of the form $[x, y]=\{z \in L: x \leq z \leq y\}$ are called order intervals or simply intervals. Clearly, a subset of $L$ is order bounded if and only if it is included in an interval.

A subset $A$ of $L$ has a least upper bound (or a supremum), $\operatorname{denoted} \sup A$, if $\sup A$ is an upper bound of $A$ and whenever $x$ is an upper bound of $A$, then $\sup A \leq x$. The greatest lower bound (or infimum) is defined analogously. Following the standard lattice terminology, the supremum and infimum of a set $\{x, y\}$ will be denoted by $x \vee y$ and $x \wedge y$, respectively. That is,

$$
x \vee y=\sup \{x, y\} \quad \text { and } \quad x \wedge y=\inf \{x, y\} .
$$

For an $m$-tuple $x_{1}, x_{2}, \ldots, x_{m}$ of vectors we shall write $\bigvee_{i=1}^{m} x_{i}$ for the supremum of the set $\left\{x_{1}, x_{2}, \ldots, x_{m}\right\}$ and $\left[\bigvee_{i=1}^{m} x_{i}\right]^{+}$for the supremum of the set $\left\{0, x_{1}, \ldots, x_{m}\right\}$. That is,

$$
\bigvee_{i=1}^{m} x_{i}=\sup \left\{x_{1}, x_{2}, \ldots, x_{m}\right\} \quad \text { and } \quad\left[\bigvee_{i=1}^{m} x_{i}\right]^{+}=\sup \left\{0, x_{1}, \ldots, x_{m}\right\} .
$$

An ordered vector space $L$ is called a Riesz space (or a vector lattice) if $x \vee y$ and $x \wedge y$ exist in $L$ for all $x, y \in L$. For extensive and detailed treatments of Riesz spaces see [10, 11] and [23].

The symbol $L^{*}$ denotes the algebraic dual of $L$, i.e., the real vector space of all linear functionals on $L$. A linear functional $f \in L^{*}$ is said to be positive if $f(x) \geq 0$ holds for all $x \in L_{+}$. The collection of all positive linear functionals on $L$ is denoted

\footnotetext{
${ }^{4}$ The inequality $x \geq y$ is also expressed by saying that $x$ dominates $y$.
} 
by $L_{+}^{*}$ and is known as the cone of positive linear functionals. Since the cone $L_{+}$ is generating, it follows that $L^{*}$ is automatically an ordered vector space by letting $f \geq g$ whenever $f-g \in L_{+}^{*}$ or $f(x) \geq g(x)$ for each $x \in L_{+}$.

A linear functional $f$ is said to be order bounded if it carries order bounded sets of $L$ to bounded subsets of $\mathbb{R}$. The collection $L^{\sim}$ of all order bounded linear functionals on $L$ is a vector subspace of $L^{*}$ and is called the order dual of $L$. Clearly, every positive linear functional is order bounded, and so every regular linear functional (i.e., every linear functional that can be written as a difference of two positive linear functionals) is likewise order bounded. If (as usual) $L^{r}$ denotes the vector space of all regular linear functionals, then we have the following vector subspace inclusions:

$$
L^{r} \subseteq L^{\sim} \subseteq L^{*}
$$

It should be noticed that if $L^{\sim}$ is a Riesz space, then $L^{r}=L^{\sim}$.

We close the section with a useful result from the work of T. Andô [15].

Lemma 2.1. Let $(L, \tau)$ be a complete metrizable ordered topological vector space such that $L_{+}$is generating and $\tau$-closed. If $\left\{U_{n}: n \in \mathbb{N}\right\}$ is a $\tau$-neighborhood base of zero consisting of $\tau$-closed circled sets such that $U_{n+1}+U_{n+1} \subseteq U_{n}$ holds for each $n$, then the countable collection $\left\{V_{n}\right\}$, where

$$
V_{n}=U_{n} \cap L_{+}-U_{n} \cap L_{+},
$$

is also a $\tau$-neighborhood base of zero.

Proof. Let $\tau_{1}$ be the linear topology of $L$ with basis $\left\{V_{n}\right\}$. By [29] Lemma 2, p. 221], $\left(L, \tau_{1}\right)$ is a complete metrizable topological vector space. Moreover, the identity operator $I:\left(L, \tau_{1}\right) \rightarrow(L, \tau)$ is continuous. But then, by the Open Mapping Theorem (see, for instance [18, Theorem 8, p. 41]), I is a homeomorphism. This implies $\tau_{1}=\tau$.

\section{The Super Order Dual of an Ordered Vector Space}

In this section, we shall introduce a collection of real-valued functions defined on the positive cone $L_{+}$of an ordered vector space $L$ that provides an extension of the topological and algebraic duals of $L$. We shall refer to this collection of real-valued functions as the super algebraic dual of $L$. Before we introduce this dual, let us isolate some properties of functions which will play an important role in our study.

Definition 3.1. A function $f: L_{+} \rightarrow \mathbb{R}$ is said to be:

1. Positive, if $f(x) \geq 0$ holds for all $x \in L_{+}$.

2. Super-additive, if $f(x)+f(y) \leq f(x+y)$ holds for all $x, y \in L_{+}$.

3. Positively homogeneous, if $f(\alpha x)=\alpha f(x)$ holds for every $\alpha \geq 0$ and all $x \in L_{+}$.

4. Monotone, if $0 \leq x \leq y$ implies $f(x) \leq f(y)$.

5. Upper order bounded, if it carries order intervals of the form $[0, x]$ to bounded from above subsets in $\mathbb{R}$.

If $f \in L^{*}$, then $\left.f\right|_{L_{+}}$(the restriction of $f$ to $L_{+}$) is additive and positively homogeneous. In particular, $\left.f\right|_{L_{+}}$is super-additive and positively homogeneous. On the other hand, if $f: L_{+} \rightarrow \mathbb{R}$ is additive and positively homogeneous, then the formula

$$
\hat{f}(x)=f\left(x_{1}\right)-f\left(x_{2}\right),
$$


where $x=x_{1}-x_{2}$ and $x_{1}, x_{2} \in L_{+}$, defines a linear functional on $L$ (i.e., $\hat{f} \in L^{*}$ )

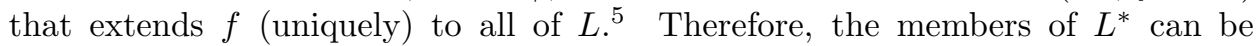
identified with the additive positively homogeneous real functions on $L_{+} 6$ This observation leads us to the definition of the super algebraic dual of $L$, which will play a central role in this paper.

Definition 3.2. The collection of all super-additive and positively homogeneous real functions defined on $L_{+}$is called the super algebraic dual of $L$ and is denoted $\mathcal{H}_{s}^{*}$. That is,

$$
\mathcal{H}_{s}^{*}=\left\{f \in \mathbb{R}^{L_{+}}: f \text { is super-additive and positively homogeneous }\right\} .
$$

We also isolate and name some important subsets of the super algebraic dual. First, we define the super dual cone $\mathcal{H}_{s}^{+}$of $L$, which is the collection of all functions in $\mathcal{H}_{s}^{*}$ that are positive. That is,

$$
\mathcal{H}_{s}^{+}=\left\{f \in \mathcal{H}_{s}^{*}: f(x) \geq 0 \text { for all } x \in L_{+}\right\} .
$$

Second, the super order dual $\mathcal{H}_{s}^{\sim}$ of $L$ is the collection of all upper order bounded functions in $\mathcal{H}_{s}^{*}$. That is,

$$
\mathcal{H}_{s}^{\sim}=\left\{f \in \mathcal{H}_{s}^{*}: f([0, x]) \text { is bounded from above in } \mathbb{R} \text { for all } x \in L_{+}\right\} .
$$

The above collections of functions will be referred to collectively as the super duals of $L$. A few basic properties of functions in the super order dual are included in the next result.

Lemma 3.3. Every function in $\mathcal{H}_{s}^{*}$ is concave and vanishes at zero.

Corollary 3.4. If $f \in \mathcal{H}_{s}^{*}$, then for each $x \in L_{+}$the upper contour set

$$
U_{f}^{x}=\{y \in X: \quad f(y) \geq f(x)\}
$$

of $f$ at $x$ is convex. Moreover, if $f \in \mathcal{H}_{s}^{+}$, then for each $x \in L_{+}$the upper contour set $U_{f}^{x}$ is convex and comprehensive, i.e., $U_{f}^{x}+L_{+} \subseteq U_{f}^{x}$.

The functions in $\mathcal{H}_{s}^{+}$are monotone.

Lemma 3.5. Every function in $\mathcal{H}_{s}^{+}$is monotone and upper order bounded.

Proof. Let $f \in \mathcal{H}_{s}^{+}$, and assume that $x, y \in L_{+}$satisfy $x \geq y$. Since $x-y \geq 0$, the super-additivity and positivity of $f$ imply

$$
f(x)=f(x-y+y) \geq f(x-y)+f(y) \geq f(y) .
$$

Thus, $f$ is monotone and $f([0, x]) \subseteq[0, f(x)]$.

Lemma 3.6. The super duals $\mathcal{H}_{s}^{+}, \mathcal{H}_{s}^{\sim}$, and $\mathcal{H}_{s}^{*}$ are all wedges in $\mathbb{R}^{L_{+}}$, and they are closed under finite pointwise infima. Moreover, $\mathcal{H}_{s}^{+}$is a convex cone in $\mathbb{R}^{L_{+}}$.

Proof. We show that $\mathcal{H}_{s}^{*}$ is a wedge and that it is closed under finite pointwise infima. The other cases are trivial. So, let $f, g \in \mathcal{H}_{s}^{*}$. Clearly, $f+g$ is positively homogeneous. Now if $x, y \in L_{+}$, then we have

$$
\begin{aligned}
(f+g)(x+y) & =f(x+y)+g(x+y) \geq[f(x)+f(y)]+[g(x)+g(y)] \\
& =[f(x)+g(x)]+[f(y)+g(y)]=(f+g)(x)+(f+g)(y) .
\end{aligned}
$$

\footnotetext{
${ }^{5}$ When $L$ is Archimedean, L. V. Kantorovich has shown that a function $f: L_{+} \rightarrow \mathbb{R}_{+}$extends to a positive linear functional on $L$ if and only if $f$ is additive; see [11, Theorem 1.7, p. 7].

${ }^{6}$ Notice here that this is possible since the cone of $L_{+}$is generating. If $L_{+}$is not generating, then our analysis must be confined to the vector subspace generated by $L_{+}$, i.e., to $L_{+}-L_{+}$.
} 
Therefore, $f+g$ is super-additive, i.e., $f+g \in \mathcal{H}_{s}^{*}$. Thus, $\mathcal{H}_{s}^{*}$ is a wedge in $\mathbb{R}^{L_{+}}$.

To see that $\mathcal{H}_{s}^{*}$ is closed under finite pointwise infima, let $f, g \in \mathcal{H}_{s}^{*}$ again, and define $h: L_{+} \rightarrow \mathbb{R}$ by $h(x)=\min \{f(x), g(x)\}$. Clearly, $h$ is positively homogeneous. If $x \in L_{+}$, then

$$
h(x)+h(y) \leq f(x)+f(y) \leq f(x+y) \quad \text { and } \quad h(x)+h(y) \leq g(x+y) .
$$

Therefore, $h(x)+h(y) \leq \min \{f(x+y), g(x+y)\}=h(x+y)$. This shows that the pointwise infimum $h$ of $f$ and $g$ belongs to $\mathcal{H}_{s}^{*}$.

Since the super duals of $L$ are subsets of $\mathbb{R}^{L_{+}}$, they inherit the natural pointwise order of $\mathbb{R}^{L_{+}}$. That is, for $f, g: L_{+} \rightarrow \mathbb{R}$ we write $f \geq g$ (and say that $f$ dominates $g$ ) whenever $f(x) \geq g(x)$ holds for each $x \in L_{+}$. We have the following characterization of domination amongst positively homogeneous functions.

Lemma 3.7. Assume that $f, g: L_{+} \rightarrow \mathbb{R}_{+}$are positively homogeneous functions. If $f\left(x_{0}\right)>0$ holds for some $x_{0} \in L_{+}$, then the following statements are equivalent.

1. The function $g$ dominates $f$, i.e., $g(y) \geq f(y)$ holds for each $y \in L_{+}$.

2. For each $y \in L_{+}$satisfying $f(y)=f\left(x_{0}\right)$ we have $g(y) \geq f\left(x_{0}\right)$, i.e.,

$$
\left\{y \in L_{+}: f(y)=f\left(x_{0}\right)\right\} \subseteq\left\{y \in L_{+}: g(y) \geq f\left(x_{0}\right)\right\} .
$$

Proof. (1) $\Longrightarrow(2)$ If $g \geq f$ and a vector $y \in L_{+}$satisfies $f(y)=f\left(x_{0}\right)$, then $g(y) \geq f(y)=f\left(x_{0}\right)$.

$(2) \Longrightarrow(1)$ Let $y \in L_{+}$. If $f(y)=0$, then $g(y) \geq f(y)$ is trivially true. Therefore, we can assume that $f(y)>0$. In this case, $f\left(\frac{f\left(x_{0}\right)}{f(y)} y\right)=f\left(x_{0}\right)$, and so our hypothesis implies $\frac{f\left(x_{0}\right)}{f(y)} g(y)=g\left(\frac{f\left(x_{0}\right)}{f(y)} y\right) \geq f\left(x_{0}\right)$ or $g(y) \geq f(y)$. So, $g(y) \geq f(y)$ holds for each $y \in L_{+}$, i.e., $g$ dominates $f$.

\section{Lattice Properties of the Super Duals}

We start by introducing some notation. If $f_{1}, \ldots, f_{m}$ are $m$ functions in $\mathcal{H}_{s}^{\sim}$, then we shall denote the supremum in $\mathcal{H}_{s}^{\sim}$ (if it exists) of the set $\left\{0, f_{1}, \ldots, f_{m}\right\}$ by $\left[\bigvee_{i=1}^{m} f_{i}\right]^{+}$, i.e., we shall write

$$
\left[\bigvee_{i=1}^{m} f_{i}\right]^{+}=\sup \left\{0, f_{1}, \ldots, f_{m}\right\}
$$

In particular, if $f \in \mathcal{H}_{s}^{\sim}$, then we have $f^{+}=f \vee 0=\sup \{0, f\}$.

We are now ready to introduce the notion of a Riesz-Kantorovich functional.

Definition 4.1. For each $i=1,2, \ldots, m$ let $f_{i}: L_{+} \rightarrow(-\infty, \infty]$ be an extended real-valued function. Then the Riesz-Kantorovich functional of the $m$-tuple $f=\left(f_{1}, f_{2}, \ldots, f_{m}\right)$ is the extended real-valued function $\mathcal{R}_{f}: L_{+} \rightarrow(-\infty, \infty]$ defined by

$$
\mathcal{R}_{f}(x)=\sup \left\{\sum_{i=1}^{m} f_{i}\left(y_{i}\right): y_{i} \in[0, x] \text { for each } i \text { and } \sum_{i=1}^{m} y_{i} \leq x\right\} .
$$

It should be immediate that if each $f_{i}$ belongs to the super order dual $\mathcal{H}_{s}^{\sim}$, then the Riesz-Kantorovich functional $\mathcal{R}_{f}$ is a positive real-valued function. In addition, it should be noticed that if one of the functions $f_{i}$ is monotone, then

$$
\mathcal{R}_{f}(x)=\sup \left\{\sum_{i=1}^{m} f_{i}\left(y_{i}\right): y_{i} \in[0, x] \text { for each } i \text { and } \sum_{i=1}^{m} y_{i}=x\right\} \text {. }
$$


In particular, if $f \in \mathcal{H}_{s}^{+}$, then Lemma 3.5 implies that

$$
\mathcal{R}_{f}(x)=\sup _{0 \leq y \leq x} f(y)=f(x)
$$

for each $x \in L_{+}$. That is, $\mathcal{R}_{f}=f$ for each $f \in \mathcal{H}_{s}^{+}$.

The Riesz-Kantorovich formula of an $m$-tuple $\left(f_{1}, \ldots, f_{m}\right)$ of functions in $\mathcal{H}_{s}^{\sim}$ is an element of $\mathcal{H}_{s}^{+}$. In fact, it is the supremum in $\mathcal{H}_{s}^{\sim}$ of the set $\left\{0, f_{1}, \ldots, f_{m}\right\}$.

Lemma 4.2. The Riesz-Kantorovich functional of an $m$-tuple $f=\left(f_{1}, f_{2}, \ldots, f_{m}\right)$ of functions in $\mathcal{H}_{s}^{\sim}$ is the supremum of the set $\left\{0, f_{1}, \ldots, f_{m}\right\}$ in $\mathcal{H}_{s}^{\sim}$. That is, the least upper bound $\left[\bigvee_{i=1}^{n} f_{i}\right]^{+}$of the set $\left\{0, f_{1}, \ldots, f_{m}\right\}$ exists in $\mathcal{H}_{s}^{\sim}$ and for each $x \in L_{+}$we have

$$
\left[\bigvee_{i=1}^{n} f_{i}\right]^{+}(x)=\mathcal{R}_{f}(x)=\sup \left\{\sum_{i=1}^{m} f_{i}\left(y_{i}\right): y_{i} \in[0, x] \text { for each } i \text { and } \sum_{i=1}^{m} y_{i} \leq x\right\} .
$$

Proof. Let $f=\left(f_{1}, f_{2}, \ldots, f_{m}\right)$ be an $m$-tuple of functions in $\mathcal{H}_{s}^{\sim}$. We shall establish first that the Riesz-Kantorovich functional $\mathcal{R}_{f}$ is positive, super-additive, and positively homogeneous. Start by observing that since each $f_{i}$ is order upper bounded and satisfies $f_{i}(0)=0$, the Riesz-Kantorovich functional $\mathcal{R}_{f}$ takes values in $\mathbb{R}_{+}$.

The positive homogeneity of $\mathcal{R}_{f}$ is a consequence of the fact that each $f_{i}$ is positively homogeneous and that for each $\alpha>0$ and $x \in L_{+}$, a vector $\left(y_{1}, \ldots, y_{m}\right)$ in $L_{+}^{m}$ satisfies $\sum_{i=1}^{m} y_{i} \leq x$ if and only if $\sum_{i=1}^{m} \alpha y_{i} \leq \alpha x$.

For the super-additivity of $\mathcal{R}_{f}$, fix $x, y \in L_{+}$, and let $\left(x_{1}, \ldots, x_{m}\right) \in L_{+}^{m}$ satisfy $\sum_{i=1}^{m} x_{i} \leq x$ and $\left(y_{1}, \ldots, y_{m}\right) \in L_{+}^{m}$ satisfy $\sum_{i=1}^{m} y_{i} \leq y$. Then, we have

$$
\left(x_{1}+y_{1}, \ldots, x_{m}+y_{m}\right) \in L_{+}^{m} \quad \text { and } \quad \sum_{i=1}^{m}\left(x_{i}+y_{i}\right) \leq x+y .
$$

Now the super-additivity of each $f_{i}$ yields

$$
\begin{aligned}
{\left[f_{1}\left(x_{1}\right)+\cdots+\right.} & \left.f_{m}\left(x_{m}\right)\right]+\left[f_{1}\left(y_{1}\right)+\cdots+f_{m}\left(y_{m}\right)\right] \\
& =\left[f_{1}\left(x_{1}\right)+f\left(y_{1}\right)\right]+\cdots+\left[f\left(x_{m}\right)+f\left(y_{m}\right)\right] \\
& \leq f_{1}\left(x_{1}+y_{1}\right)+\cdots+f_{m}\left(x_{m}+y_{m}\right) \\
& \leq \mathcal{R}_{f}(x+y) .
\end{aligned}
$$

This easily implies $\mathcal{R}_{f}(x)+\mathcal{R}_{f}(y) \leq \mathcal{R}_{f}(x+y)$. Therefore, $\mathcal{R}_{f}$ is super-additive, and thus $\mathcal{R}_{f} \in \mathcal{H}_{s}^{+}$.

Next, it should be obvious that $\mathcal{R}_{f}(x) \geq f_{i}(x)$ holds for all $x \in L_{+}$and each $i$. That is, $\mathcal{R}_{f}$ is an upper bound in $\mathcal{H}_{s}^{\sim}$ for the set $\left\{0, f_{1}, \ldots, f_{m}\right\}$. To see that $\mathcal{R}_{f}$ is the least upper bound of the set $\left\{0, f_{1}, \ldots, f_{m}\right\}$ in $\mathcal{H}_{s}^{\sim}$, assume that some $g \in \mathcal{H}_{s}^{\sim}$ satisfies $g \geq 0$ and $g \geq f_{i}$ for $i=1, \ldots, m$. If $x \in L_{+}$and $\left(y_{1}, \ldots, y_{m}\right) \in L_{+}^{m}$ satisfies $\sum_{i=1}^{m} y_{i} \leq x$, then, using the super-additivity and monotonicity of $g$ (see Lemma [.5.), we obtain

$$
\sum_{i=1}^{m} f_{i}\left(y_{i}\right) \leq \sum_{i=1}^{m} g\left(y_{i}\right) \leq g\left(\sum_{i=1}^{m} y_{i}\right) \leq g(x) .
$$

This clearly implies $\mathcal{R}_{f}(x) \leq g(x)$. Consequently, $\mathcal{R}_{f}(x) \leq g(x)$ for each $x \in L_{+}$, and thus $\mathcal{R}_{f}=\sup \left\{0, f_{1}, \ldots, f_{m}\right\}$ in $\mathcal{H}_{s}^{\sim}$. 
Remarkably, the supremum of any $m$-tuple of order bounded linear functionals in $\mathcal{H}_{s}^{\sim}$ exists. This is a special case of the following result.

Theorem 4.3. If $f_{1}, \ldots, f_{m}$ are functions in $\mathcal{H}_{s}^{\sim}$ and at least one of them either is positive or belongs to $L^{\sim}$, then the supremum $\bigvee_{i=1}^{m} f_{i}$ exists in $\mathcal{H}_{s}^{\sim}$ and is given by the Riesz-Kantorovich formula. That is, for each $x \in L_{+}$we have

$$
\left[\bigvee_{i=1}^{m} f_{i}\right](x)=\sup \left\{\sum_{i=1}^{m} f_{i}\left(y_{i}\right): y_{i} \in[0, x] \text { for each } i \text { and } \sum_{i=1}^{m} y_{i}=x\right\}
$$

Proof. By Lemma 4.2, we know that $\sup \left\{0, f_{1}, \ldots, f_{m}\right\}=\mathcal{R}_{f}$ in $\mathcal{H}_{s}^{\sim}$. Now assume that $f_{j}$ is positive for some $j$. Then, $\sup \left\{0, f_{1}, \ldots, f_{m}\right\}=\sup \left\{f_{1}, \ldots, f_{m}\right\}$ is clearly true, and therefore

$$
\left[\bigvee_{i=1}^{m} f_{i}\right](x)=\sup \left\{\sum_{i=1}^{m} f_{i}\left(y_{i}\right): y_{i} \in[0, x] \text { for each } i \text { and } \sum_{i=1}^{m} y_{i} \leq x\right\} .
$$

Now the validity of $(\star)$ easily follows from the monotonicity of $f_{j}$.

Next, assume that $f_{i} \in L^{\sim}$ holds for some $i$; we can suppose that $f_{1} \in L^{\sim}$. Then, each $f_{i}-f_{1}$ belongs to $\mathcal{H}_{s}^{\sim}$. Let $g=\left(f_{2}-f_{1}, f_{3}-f_{1}, \ldots, f_{m}-f_{1}\right)$ and consider the Riesz-Kantorovich functional $\mathcal{R}_{g}$. From Lemma 4.2 it follows that

$$
\mathcal{R}_{g}=\left[\bigvee_{i=2}^{m}\left(f_{i}-f_{1}\right)\right]^{+}
$$

Since $\mathcal{H}_{s}^{\sim}$ is a wedge, we have $\mathcal{R}_{g}+f_{1} \in \mathcal{H}_{s}^{\sim}$. Clearly, $\mathcal{R}_{g}+f_{1} \geq f_{1}$. Now let $x \in L_{+}$, and note that for each $2 \leq j \leq m$ we have

$$
\begin{aligned}
\mathcal{R}_{g}(x)+f_{1}(x) & =\sup \left\{\sum_{i=2}^{m}\left(f_{i}-f_{1}\right)\left(y_{i}\right): y \in[0, x]^{m-1} \text { and } \sum_{i=2}^{m} y_{i} \leq x\right\}+f_{1}(x) \\
& \geq\left(f_{j}-f_{1}\right)(x)+f_{1}(x)=f_{j}(x) .
\end{aligned}
$$

Therefore, $\mathcal{R}_{g}+f_{1}$ is an upper bound of the set $\left\{f_{1}, \ldots, f_{m}\right\}$ in $\mathcal{H}_{s}^{\sim}$.

Now we shall prove that $\mathcal{R}_{g}+f_{1}$ is the least upper bound of the set $\left\{f_{1}, \ldots, f_{m}\right\}$ in $\mathcal{H}_{s}^{\sim}$. To this end, suppose that some $h \in \mathcal{H}_{s}^{\sim}$ dominates each $f_{i}$. Once again, $h-f_{1} \in \mathcal{H}_{s}^{\sim}$. Furthermore, $h-f_{1} \geq 0$ and $h-f_{1}$ dominates $f_{i}-f_{1}$ for $2 \leq i \leq m$. Therefore, by Lemma $4.2 \mathcal{R}_{g} \leq h-f_{1}$, and so $\mathcal{R}_{g}+f_{1} \leq h$. This shows that $\mathcal{R}_{g}+f_{1}$ is the least upper bound in $\mathcal{H}_{s}^{\sim}$ of the set $\left\{f_{1}, \ldots, f_{m}\right\}$.

Finally, it easily follows from the additivity of $f_{1}$ that for each $x \in L_{+}$we have

$$
\left[\bigvee_{i=1}^{m} f_{i}\right](x)=\left(\mathcal{R}_{g}+f_{1}\right)(x)=\left\{\sum_{i=1}^{m} f_{i}\left(y_{i}\right): y_{i} \in[0, x] \text { for each } i \text { and } \sum_{i=1}^{m} y_{i}=x\right\} \text {. }
$$

This completes the proof.

Corollary 4.4. The set $\mathcal{H}_{s}^{+}$equipped with the pointwise ordering is a lattice. Moreover, for any pair $f_{1}, f_{2} \in \mathcal{H}_{s}^{+}$and any $x \in L_{+}$we have

$$
\begin{aligned}
& {\left[f_{1} \vee f_{2}\right](x)=\sup \left\{f_{1}\left(y_{1}\right)+f_{2}\left(y_{2}\right): y_{1}, y_{2} \in L_{+} \text {and } y_{1}+y_{2}=x\right\},} \\
& {\left[f_{1} \wedge f_{2}\right](x)=\min \left\{f_{1}(x), f_{2}(x)\right\} .}
\end{aligned}
$$


It should be noticed that the finite suprema in $\mathcal{H}_{s}^{+}$need not coincide with the pointwise suprema in $\mathbb{R}^{L_{+}}$. For instance, if we let $L=\mathbb{R}^{2}$ and $L_{+}=\mathbb{R}_{+}^{2}$, and consider the "linear" functions $f_{1}, f_{2} \in \mathcal{H}_{s}^{+}$defined by

$$
f_{1}(x, y)=x \quad \text { and } \quad f_{2}(x, y)=y \text {, }
$$

then $\mathcal{R}_{\left(f_{1}, f_{2}\right)}(x, y)=x+y$, while the pointwise supremum of $f_{1}$ and $f_{2}$ in $\mathbb{R}^{L_{+}}$ satisfies

$$
\max \left\{f_{1}(x, y), f_{2}(x, y)\right\}=\max \{x, y\},
$$

which is different than $\mathcal{R}_{\left(f_{1}, f_{2}\right)}$. This phenomenon is analogous to the idea of a lattice-subspace; see [26, 27]. Moreover, notice that Theorem 4.3 does not tell us that the vector space $\mathcal{H}_{s}^{+}-\mathcal{H}_{s}^{+}$is a vector lattice under the partial ordering induced by $\mathcal{H}_{s}^{+}$. In fact, $\left(\mathcal{H}_{s}^{+}-\mathcal{H}_{s}^{+}\right) \cap \mathbb{R}^{L+} \neq \mathcal{H}_{s}^{+}$, and the order induced by the cone $\mathcal{H}_{s}^{+}$ is not the pointwise ordering of $\mathbb{R}^{L+}$.

\section{Embedding Duals in the Super Order Dual}

Having established that the super dual cone is a lattice, we are now interested in the circumstances under which the canonical embedding of the duals in the super dual preserves suprema. To this end, assume from now to the end of the paper that $L$ is also an ordered topological vector space. That is, $L$ is also equipped with a Hausdorff locally convex topology $\tau$ for which $L_{+}$is $\tau$-closed and every order interval is $\tau$-bounded.

As usual, $L^{\prime}$ denotes the topological dual of $(L, \tau)$ and $L_{+}^{\prime}$ denotes the collection of all positive $\tau$-continuous linear functionals on $L$, i.e.,

$$
L_{+}^{\prime}=\left\{f \in L^{\prime}: \quad f(x) \geq 0 \text { holds for each } x \in L_{+}\right\} .
$$

Since we assume that the cone $L_{+}$is generating, it follows that $L_{+}^{\prime}$ is a $\sigma\left(L^{\prime}, L\right)$ closed cone of $L^{\prime}$. Moreover, since the order intervals of $L$ are $\tau$-bounded, it should be clear that $L^{\prime} \subseteq L^{\sim}$.

We now isolate and name the collection of all upper $\tau$-semicontinuous functions 7 of $\mathcal{H}_{s}^{*}$.

Definition 5.1. The super topological dual $\mathcal{H}_{s}^{\prime}$ of $L$ is the collection of all superadditive, positively homogeneous, and upper $\tau$-semicontinuous functions from $L_{+}$ to $\mathbb{R}$. That is,

$$
\mathcal{H}_{s}^{\prime}=\left\{f \in \mathcal{H}_{s}^{*}: f \text { is upper } \tau \text {-semicontinuous }\right\} .
$$

The subset of all positive functions in $\mathcal{H}_{s}^{\prime}$ will be denoted by $\left(\mathcal{H}_{s}^{\prime}\right)_{+}$.

The super topological dual $\mathcal{H}_{s}^{\prime}$ is closed under finite pointwise infima. Indeed, since for any family of functions $\left\{f_{i}\right\}_{i \in I}$, where $f_{i}: X \rightarrow[-\infty, \infty]$, and any $c \in \mathbb{R}$ we have

$$
\left\{x \in X: \inf _{i \in I} f(x) \geq c\right\}=\bigcap_{i \in I}\left\{x \in X: f_{i}(x) \geq c\right\},
$$

\footnotetext{
${ }^{7}$ Recall that a function $f: X \rightarrow \mathbb{R}$ on a topological space is said to be upper semicontinuous if for each $c \in \mathbb{R}$ the set $\left\{x \in L_{+}: f(x) \geq c\right\}$ is closed. It is well known that a function $f: X \rightarrow \mathbb{R}$ is upper $\tau$-semicontinuous if and only if $x_{\alpha} \rightarrow x$ in $X$ implies $\limsup _{\alpha} f\left(x_{\alpha}\right) \leq f(x)$; see [7] Lemma 2.39, p. 43].
} 
it follows that the pointwise infimum of a family of upper $\tau$-semicontinuous functions is an upper $\tau$-semicontinuous function - which is also super-additive and positively homogeneous if each $f_{i}$ is likewise so.

The next result characterizes the functions in $\left(\mathcal{H}_{s}^{\prime}\right)_{+}$that are upper $\tau$-semicontinuous.

Lemma 5.2. A function $f \in \mathcal{H}_{s}^{+}$is upper $\tau$-semicontinuous, i.e., $f \in\left(\mathcal{H}_{s}^{\prime}\right)_{+}$, if and only if for each $x \in L_{+}$we have

$$
f(x)=\inf \left\{h(x): h \in L^{\prime} \text { and } h \geq f\right\} .
$$

Proof. Fix a function $f \in \mathcal{H}_{s}^{+}$. If for each $x \in L_{+}$we have

$$
f(x)=\inf \left\{h(x): h \in L^{\prime} \text { and } h \geq f\right\},
$$

then $f$ is clearly upper $\tau$-semicontinuous; see the discussion following Definition 5.1

For the converse, suppose that $f$ is upper $\tau$-semicontinuous. Let $x_{0} \in L_{+}$and fix $\epsilon>0$. We must establish that there exists some $h \in L^{\prime}$ satisfying $h \geq f$ and $f\left(x_{0}\right) \leq h\left(x_{0}\right)<f\left(x_{0}\right)+\epsilon$.

To this end, assume first that $f\left(x_{0}\right)>0$. Put $\alpha=\frac{f\left(x_{0}\right)}{f\left(x_{0}\right)+\epsilon}$ and notice that $0<\alpha<1$. By the positive homogeneity of $f$ we have $f\left(\alpha x_{0}\right)=\alpha f\left(x_{0}\right)<f\left(x_{0}\right)$. That is, $\alpha x_{0} \notin U_{f}^{x_{0}}$, where $U_{f}^{x_{0}}$ is the upper contour set of $f$ at $x_{0}$, i.e., $U_{f}^{x_{0}}=$ $\left\{x \in L_{+}: f(x) \geq f\left(x_{0}\right)\right\}$. Since $U_{f}^{x_{0}}$ is $\tau$-closed and convex, there exist (by the Separation Theorem) a $\tau$-continuous linear functional $g$ and a real number $\eta$ satisfying $g\left(\alpha x_{0}\right)<\eta$ and $g(x)>\eta$ for each $x \in U_{f}^{x_{0}}$. The comprehensiveness of the set $U_{f}^{x_{0}}$ (see Corollary 3.7) guarantees that $g$ is also a positive linear functional. This implies that $\eta>g\left(\alpha x_{0}\right) \geq 0$. Letting $h=\frac{f\left(x_{0}\right)}{\eta} g \in L^{\prime}$, we note that $h(y)=\frac{f\left(x_{0}\right)}{\eta} g(y)>f\left(x_{0}\right)$ for each $y \in U_{f}^{x_{0}}$. By Lemma [3.7 we get $h \geq f$. Finally, notice that $h\left(\alpha x_{0}\right)=\frac{f\left(x_{0}\right)}{\eta} g\left(\alpha x_{0}\right)<f\left(x_{0}\right)$ implies $h\left(x_{0}\right)<\frac{f\left(x_{0}\right)}{\alpha}=f\left(x_{0}\right)+\epsilon$. This completes the proof when $f\left(x_{0}\right)>0$.

We now consider the case $f\left(x_{0}\right)=0$. If $f=0$, then $f$ is linear and the conclusion holds trivially. Thus, it remains to consider the case where $f(y)>0$ for some $y \in L_{+}$. For each $0<\alpha<1$ let $z_{\alpha}=\alpha x_{0}+(1-\alpha) y$. Since $f$ is super-additive and positively homogeneous, it must be the case that $f\left(z_{\alpha}\right)>0$ for each $0<\alpha<1$. From $\lim _{\alpha \uparrow 1} z_{\alpha}=x_{0}$ and the upper $\tau$-semicontinuity of $f$, it follows that $0 \leq$ $\lim \sup _{\alpha \uparrow 1} f\left(z_{\alpha}\right) \leq f\left(x_{0}\right)=0$. This implies $\lim _{\alpha \uparrow 1} f\left(z_{\alpha}\right)=0$. In particular, there exists some $\frac{1}{2} \leq \alpha_{0}<1$ such that $0<f\left(z_{\alpha}\right)<\frac{\epsilon}{2}$ for all $\alpha_{0} \leq \alpha<1$. According to the preceding case, for each $\alpha_{0} \leq \alpha<1$ there exists some $h_{\alpha} \in L^{\prime}$ satisfying $h_{\alpha} \geq f \geq 0$ and $0<f\left(z_{\alpha}\right) \leq h_{\alpha}\left(z_{\alpha}\right)<\frac{\epsilon}{2}$. Now observe that for each $\alpha_{0} \leq \alpha<1$ we have

$$
\frac{1}{2} h_{\alpha}\left(x_{0}\right) \leq \alpha h_{\alpha}\left(x_{0}\right) \leq \alpha h_{\alpha}\left(x_{0}\right)+(1-\alpha) h_{\alpha}(y)=h_{\alpha}\left(z_{\alpha}\right)<\frac{\epsilon}{2},
$$

and so $f\left(x_{0}\right) \leq h_{\alpha}\left(x_{0}\right)<\epsilon$ holds for all $\alpha_{0} \leq \alpha<1$, and the proof is finished.

The next result asserts that $\mathcal{H}_{s}^{\prime} \subseteq \mathcal{H}_{s}^{\sim}$. That is, every super-additive, positively homogeneous, and upper $\tau$-semicontinuous function from $L_{+}$to $\mathbb{R}$ is upper order bounded 8

\footnotetext{
${ }^{8}$ Note that since for each function $f \in \mathcal{H}_{s}^{*}$ the sets $\left\{x \in L_{+}: f(x) \geq c\right\}$ are all convex, it follows that if $f$ is upper $\tau$-semicontinuous, then $f$ is also upper semicontinuous for every locally convex topology on $L$ that is consistent with the dual pair $\left\langle L, L^{\prime}\right\rangle$.
} 
Lemma 5.3. Every function in $\mathcal{H}_{s}^{\prime}$ carries $\tau$-bounded subsets of $L$ to bounded from above subsets in $\mathbb{R}$. In particular, we have $\mathcal{H}_{s}^{\prime} \subseteq \mathcal{H}_{s}^{\sim}$.

Proof. Suppose by way of contradiction that some $f \in \mathcal{H}_{s}^{\prime}$ carries a $\tau$-bounded subset $B$ of $L$ to an unbounded from above subset of $\mathbb{R}$. This means that there exists a sequence $\left\{x_{n}\right\} \subseteq B$ such that $f\left(x_{n}\right)>n$ holds for every $n$. Since $B$ is $\tau$-bounded, we have $\frac{1}{n} x_{n} \stackrel{\tau}{\rightarrow} 0$. But then, the upper $\tau$-semicontinuity and positive homogeneity of $f$ imply

$$
0=f(0) \geq \limsup _{n \rightarrow \infty} f\left(\frac{1}{n} x_{n}\right)=\limsup _{n \rightarrow \infty} \frac{1}{n} f\left(x_{n}\right) \geq 1,
$$

which is impossible.

There is a natural mapping $J: L^{*} \rightarrow \mathbb{R}^{L_{+}}$defined by

$$
J(f)(x)=\left(\left.f\right|_{L_{+}}\right)(x)=f(x)
$$

for all $x \in L_{+}$. That is, $J(f)=\left.f\right|_{L_{+}}$, the restriction of $f$ to $L_{+}$. It is easy to see that this is a linear mapping that preserves the order, i.e., $f \leq g$ in $L^{*}$ implies $J(f) \leq J(g)$. In other words, we have the following.

Lemma 5.4. The mapping $J: L^{*} \rightarrow \mathbb{R}^{L_{+}}$is an injective (i.e., one-to-one) positive operator. Moreover, $J$ maps $L^{*}$ into $\mathcal{H}_{s}^{*}, L_{+}^{*}$ into $\mathcal{H}_{s}^{+}$, and $L^{r}$ and $L^{\sim}$ into $\mathcal{H}_{s}^{\sim}$.

It is easy to see that $\left(\mathcal{H}_{s}^{\prime}\right)_{+} \subseteq \mathcal{H}_{s}^{+}$and that the operator $J: L^{*} \rightarrow \mathbb{R}^{L_{+}}$carries $L^{\prime}$ into $\mathcal{H}_{s}^{\prime}$. We are now in a position to show that the embedding $J: L^{\prime} \rightarrow \mathcal{H}_{s}^{\prime}$ also preserves finite suprema. Notice that we have yet to establish sufficient conditions for the suprema in $\mathcal{H}_{s}^{\prime}$ to coincide with suprema in $\mathcal{H}_{s}^{\sim}$, i.e., the suprema in $\mathcal{H}_{s}^{\prime}$ need not satisfy the Riesz-Kantorovich formula.

Theorem 5.5 (The Embedding Theorem). If the supremum $\bigvee_{i=1}^{m} f_{i}$ of the continuous linear functionals $f_{1}, f_{2}, \ldots, f_{m}$ on $L$ exists in $L^{\prime}$, then $\bigvee_{i=1}^{m} J\left(f_{i}\right)$ exists in $\mathcal{H}_{s}^{\prime}$ and

$$
J\left(\bigvee_{i=1}^{m} f_{i}\right)=\bigvee_{i=1}^{m} J\left(f_{i}\right) .
$$

That is, the embedding $J: L^{\prime} \rightarrow \mathcal{H}_{s}^{\prime}$ preserves finite suprema.

Proof. Let $f_{1}, \ldots, f_{m} \in L^{\prime}$ and assume that $g=\bigvee_{i=1}^{m} f_{i}$ exists in $L^{\prime}$. Identifying each $f \in L^{\prime}$ with its image $J(f)=\left.f\right|_{L_{+}}$in $\mathcal{H}_{s}^{\prime}$, what needs to be shown is that if $h \in \mathcal{H}_{s}^{\prime}$ satisfies $h \geq f_{i}$ for each $i=1, \ldots, m$, then $h \geq g$. So, assume that some function $h \in \mathcal{H}_{s}^{\prime}$ satisfies $h \geq f_{i}$ for each $i$.

Replacing each $f_{i}$ with $f_{i}-f_{1}, h$ with $h-f_{1} \in\left(\mathcal{H}_{s}^{\prime}\right)_{+}$, and $g$ with $g-f_{1}=$ $\bigvee_{i=1}^{m}\left(f_{i}-f_{1}\right) \in L_{+}^{\prime}$, we can assume without loss of generality that $f_{1}=0$. Clearly, $g \geq 0$ and $h \geq 0$.

Fix a vector $x \in L_{+}$. From Lemma 5.2, for each $\epsilon>0$ there exists some $\phi_{\epsilon} \in L^{\prime}$ satisfying $\phi_{\epsilon} \geq h$ and $\phi_{\epsilon}(x)<h(x)+\epsilon$. Clearly, $\phi_{\epsilon} \geq f_{i}$ for each $i$. Since $g$ is the supremum of the set $\left\{f_{1}, \ldots, f_{m}\right\}$ in $L^{\prime}$, we infer that $\phi_{\epsilon} \geq g$. Therefore, $g(x) \leq \phi_{\epsilon}(x)<h(x)+\epsilon$. This implies $g(x)<h(x)+\epsilon$ for every $\epsilon>0$. Consequently, $g(x) \leq h(x)$ for each $x \in L_{+}$, as desired. 


\section{The Embedding And the Riesz-Kantorovich Formula}

In this section, we turn our attention to the algebraic dual $\left(L^{\prime}\right)^{*}$ of $L^{\prime}$. Recall that every vector $x \in L$ can be considered as a vector in $\left(L^{\prime}\right)^{*}$ by letting $x(h)=h(x)$ for each $h \in L^{\prime}$. Also, if $x, y \in\left(L^{\prime}\right)^{*}$, then $x \geq y$ means that $x(h) \geq y(h)$ for all $h \in L_{+}^{\prime}$. Thus, for each $x \in L_{+}$, besides the order interval [0,x] in $L$, there is another "order interval" associated with $x$ in $\left(L^{\prime}\right)^{*}$. This is the order interval

$$
\llbracket 0, x \rrbracket=\left\{h \in\left(L^{\prime}\right)^{*}: 0 \leq h \leq x\right\} .
$$

Clearly $[0, x] \subseteq \llbracket 0, x \rrbracket$ holds for each $x \in L_{+} 9$

Now, if $f=\left(f_{1}, f_{2}, \ldots, f_{m}\right)$ is an $m$-tuple of $\tau$-continuous linear functionals on $L$, i.e., $f_{i} \in L^{\prime}$ for each $i$, then we can define another generalized Riesz-Kantorovich function $\mathcal{R}_{f}^{*}: L_{+} \rightarrow[0, \infty]$ via the formula

$$
\mathcal{R}_{f}^{*}(x)=\sup \left\{\sum_{i=1}^{m} h_{i}\left(f_{i}\right): h_{i} \in \llbracket 0, x \rrbracket \text { for each } i \text { and } \sum_{i=1}^{m} h_{i} \leq x\right\} .
$$

Clearly, $\mathcal{R}_{f}^{*}$ is positive, super-additive, and positively homogeneous. Therefore, if $\mathcal{R}_{f}^{*}$ is also real valued, then $\mathcal{R}_{f}^{*} \in \mathcal{H}_{s}^{+}$.

Lemma 6.1. If $f=\left(f_{1}, f_{2}, \ldots, f_{m}\right)$ is an $m$-tuple of $\tau$-continuous linear functionals on $L$, then for each $x \in L_{+}$we have $\mathcal{R}_{f}(x) \leq \mathcal{R}_{f}^{*}(x)$. Moreover, if some $g \in L_{+}^{\prime}$ satisfies $g \geq f_{i}$ for each $i$, then

$$
\mathcal{R}_{f}(x) \leq \mathcal{R}_{f}^{*}(x) \leq g(x)
$$

for every $x \in L_{+}$(and hence, in this case, $\mathcal{R}_{f}^{*} \in \mathcal{H}_{s}^{+}$).

Proof. Fix $x \in L_{+}$and let $g \in L_{+}^{\prime}$ satisfy $g \geq f_{i}$ for each $i$. The inequality $\mathcal{R}_{f}(x) \leq \mathcal{R}_{f}^{*}(x)$ follows immediately from the inclusion $[0, x] \subseteq \llbracket 0, x \rrbracket$. Now assume that the vector $\left(h_{1}, \ldots, h_{m}\right)$ satisfies $h_{i} \in \llbracket 0, x \rrbracket$ for each $i$ and $\sum_{i=1}^{m} h_{i} \leq x$. Since $g$ dominates each $f_{i}$, we get

$$
\sum_{i=1}^{m} h_{i}\left(f_{i}\right) \leq \sum_{i=1}^{m} h_{i}(g)=\left[\sum_{i=1}^{m} h_{i}\right](g) \leq x(g)=g(x)<\infty .
$$

This implies that $\mathcal{R}_{f}^{*}(x) \leq g(x)<\infty$, and completes the proof.

The next result informs us when the finite supremum of an $m$-tuple of continuous linear functionals satisfies the Riesz-Kantorovich formula.

Theorem 6.2. Assume that the supremum $g=\left[\bigvee_{i=1}^{m} f_{i}\right]^{+}$of an $m$-tuple of $\tau$ continuous linear functionals $f=\left(f_{1}, f_{2}, \ldots, f_{m}\right)$ exists in $L^{\prime}$. Then we have:

1. $\left[\bigvee_{i=1}^{m} f_{i}\right]^{+}=\mathcal{R}_{f}$ if and only if $\mathcal{R}_{f} \in \mathcal{H}_{s}^{\prime}$.

2. $\left[\bigvee_{i=1}^{m} f_{i}\right]^{+}=\mathcal{R}_{f}^{*}$ if and only if $\mathcal{R}_{f}^{*} \in \mathcal{H}_{s}^{\prime}$.

Proof. (1) If $g(x)=\mathcal{R}_{f}(x)$ holds for all $x \in L_{+}$, then $\mathcal{R}_{f}$ is $\tau$-continuous on $L_{+}$, and hence $\mathcal{R}_{f}$ is also upper $\tau$-semicontinuous. For the converse, assume that $\mathcal{R}_{f}$ is upper $\tau$-semicontinuous, that is, $\mathcal{R}_{f} \in \mathcal{H}_{s}^{\prime}$. From the Embedding Theorem 5.5 we know that $g$ is the supremum of the set $\left\{0, f_{1}, f_{2}, \ldots, f_{m}\right\}$ in $\mathcal{H}_{s}^{\prime}$. Also, from Lemma 4.2, we know that $\mathcal{R}_{f}$ is the supremum of the set $\left\{0, f_{1}, f_{2}, \ldots, f_{m}\right\}$ in $\mathcal{H}_{s}^{\sim}$.

\footnotetext{
${ }^{9}$ Note that the collection $\left(L^{\prime}\right)_{+}^{*}=\left\{f \in\left(L^{\prime}\right)^{*}: f \geq 0\right\}$ of all positive linear functionals on $L^{\prime}$ is a wedge of $\left(L^{\prime}\right)^{*}$, which is not necessarily a cone. However, when the cone $L_{+}^{\prime}$ is generating, $\left(L^{\prime}\right)_{+}^{*}$ is a cone in $\left(L^{\prime}\right)^{*}$.
} 
Since $\mathcal{H}_{s}^{\prime} \subseteq \mathcal{H}_{s}^{\sim}$ and $\mathcal{R}_{f}$ is upper $\tau$-semicontinuous, it follows that $\mathcal{R}_{f}$ is also the supremum of the set $\left\{0, f_{1}, f_{2}, \ldots, f_{m}\right\}$ in $\mathcal{H}_{s}^{\prime}$. This implies $g=\mathcal{R}_{f}$.

(2) Assume first that $\mathcal{R}_{f}^{*}$ is upper $\tau$-semicontinuous. As in the proof of part (1) of Lemma 4.2, we see that $\mathcal{R}_{f}^{*} \in \mathcal{H}_{s}^{+}$. Moreover, our assumption implies that $\mathcal{R}_{f}^{*} \in\left(\mathcal{H}_{s}^{\prime}\right)_{+}$. By the Embedding Theorem 5.5 it must be the case that $g \leq \mathcal{R}_{f}^{*}$. That is, $g(x) \leq \mathcal{R}_{f}^{*}(x)$ for all $x \in L_{+}$. Now a glance at Lemma 6.1 tells us that $g(x)=\mathcal{R}_{f}^{*}(x)$ for every $x \in L_{+}$. If, on the other hand, $g(x)=\mathcal{R}_{f}^{*}(x)$ for every $x \in L_{+}$, then clearly $\mathcal{R}_{f}^{*}$ is $\tau$-continuous - and hence it is also upper $\tau$ semicontinuous.

Surprisingly, when $\mathcal{R}_{f}$ is upper semicontinuous it coincides with $\mathcal{R}_{f}^{*}$.

Lemma 6.3. If $f=\left(f_{1}, f_{2}, \ldots, f_{m}\right)$ is an m-tuple of $\tau$-continuous linear functionals on $L$ and $\mathcal{R}_{f}$ is upper $\tau$-semicontinuous, then $\mathcal{R}_{f}=\mathcal{R}_{f}^{*}$.

Proof. Take an arbitrary $x \in L_{+}$. We claim that $\mathcal{R}_{f}^{*}(x)=\mathcal{R}_{f}(x)$. To see this, suppose by way of contradiction that $\mathcal{R}_{f}^{*}(x)>\mathcal{R}_{f}(x)$. By Lemma 5.2, there exists some $g \in L_{+}^{\prime}$ satisfying $g \geq \mathcal{R}_{f}$ and $\mathcal{R}_{f}^{*}(x)>g(x)$. However, since $g \geq f_{i}$ holds for each $i$, it follows from Lemma 6.1 that $\mathcal{R}_{f}^{*}(x) \leq g(x)$, which is a contradiction. Therefore, $\mathcal{R}_{f}^{*}(x)=\mathcal{R}_{f}(x)$.

Recall that if $L$ is a locally convex-solid Riesz space, then the order intervals in $L$ are $\sigma\left(L^{\prime \prime}, L^{\prime}\right)$-dense in the order intervals of $L^{\prime \prime}$ (see [11, Lemma 11.12, p. 169]). An analogous property holds whenever the Riesz-Kantorovich functionals of functions in $L^{\prime}$ are upper $\tau$-semicontinuous.

Theorem 6.4. If $\mathcal{R}_{f}$ is upper $\tau$-semicontinuous for every $f \in L^{\prime}$, then each order interval $[0, x]$ is $\sigma\left(\left(L^{\prime}\right)^{*}, L^{\prime}\right)$-dense in $\llbracket 0, x \rrbracket$.

Proof. Fix $x_{0} \in L_{+}$and suppose by way of contradiction that there exists some $z_{0} \in \llbracket 0, x_{0} \rrbracket$ satisfying $z_{0} \notin \overline{\left[0, x_{0}\right]}$, where the bar denotes $\sigma\left(\left(L^{\prime}\right)^{*}, L^{\prime}\right)$-closure. Since $\sigma\left(\left(L^{\prime}\right)^{*}, L^{\prime}\right)$ is locally convex, it follows from the Separation Theorem that there exist a linear functional $f \in L^{\prime}$ and a constant $c$ such that $f\left(z_{0}\right)>c$ and $f(x)<c$ for all $x \in\left[0, x_{0}\right]$. Therefore, $\mathcal{R}_{f}\left(x_{0}\right) \leq c$. On the other hand, by Lemma 6.3 we have $\mathcal{R}_{f}\left(x_{0}\right)=\mathcal{R}_{f}^{*}\left(x_{0}\right) \geq f\left(z_{0}\right)>c>0$, a contradiction. This contradiction establishes that $[0, x]$ is $\sigma\left(\left(L^{\prime}\right)^{*}, L^{\prime}\right)$-dense in $\llbracket 0, x \rrbracket$.

We close this section by showing that the continuity of the Riesz-Kantorovich functionals at zero is equivalent to the identity $L^{r}=L^{\sim}$.

Theorem 6.5. Every order bounded linear functional is regular (i.e., $L^{r}=L^{\sim}$ ) if and only if for every $f \in\left(L^{\sim}\right)^{m}$ its Riesz-Kantorovich functional $\mathcal{R}_{f}$ is continuous at zero for some Hausdorff locally convex topology on L.

Proof. Assume first that $L^{r}=L^{\sim}$. From $L^{\prime} \subseteq L^{\sim}$, we know that $\sigma\left(L, L^{\sim}\right)$ is a Hausdorff locally convex topology on $L$. Suppose by way of contradiction that for some $m$-tuple $f=\left(f_{1}, f_{2}, \ldots, f_{m}\right)$ of linear functionals in $L^{\sim}$ the Riesz-Kantorovich functional $\mathcal{R}_{f}$ is not $\sigma\left(L, L^{\sim}\right)$-continuous at zero. This means that there exist a net $\left\{x_{\alpha}\right\} \subseteq L_{+}$and some $c>0$ such that $x_{\alpha} \stackrel{\sigma(L, L \sim)}{\longrightarrow} 0$ while $\mathcal{R}_{f}\left(x_{\alpha}\right) \geq c>0$ holds for every $\alpha$. Since $L^{r}=L^{\sim}$, there exists some $g \in L_{+}^{\sim} \subseteq L^{\sim}$ that dominates $f_{1}, f_{2}, \ldots, f_{m}$, and therefore $\mathcal{R}_{f}(x) \leq g(x)$ for every $x \in L_{+}$. This implies that $g\left(x_{\alpha}\right) \geq c>0$ holds for each $\alpha$. This contradicts the fact that $g \in L^{\sim}$ and $x_{\alpha} \stackrel{\sigma(L, L \sim)}{\longrightarrow} 0$. 
For the converse, suppose that for every $f \in\left(L^{\sim}\right)^{m}$ the Riesz-Kantorovich functional $\mathcal{R}_{f}$ is continuous at zero for some Hausdorff locally convex topology $\tau_{f}$ on $L$. Since $L^{r} \subseteq L^{\sim}$ is always true, it suffices to show that for any $f \in L^{\sim}$ there exists a positive linear functional $g$ such that $g \geq f$. To this end, fix $f \in L^{\sim}$. If $f \leq 0$, then we are done. So, we can assume that there exists some $x \in L_{+}$such that $f(x)>0$. Clearly, $\mathcal{R}_{f}(x)>0$. Since $\mathcal{R}_{f}$ is $\tau_{f}$-continuous at zero, it follows that zero does not belong to the $\tau_{f}$-closure of $U_{\mathcal{R}_{f}}^{x}=\left\{y \in L_{+}: \mathcal{R}_{f}(y) \geq \mathcal{R}_{f}(x)\right\}$. By the Separation Theorem, there exist a non-zero $\tau_{f}$-continuous linear functional $h$ and a real number $c>0$ such that $z \in U_{\mathcal{R}_{f}}^{x}$ implies $h(z) \geq c$. Since $U_{\mathcal{R}_{f}}^{x}$ is comprehensive, it must be the case that $h \geq 0$; and so $h \in L^{\sim}$. From $\frac{\mathcal{R}_{f}(x)}{c} h(y) \geq \mathcal{R}_{f}(x)$ for each $y \in U_{\mathcal{R}_{f}}^{x}$ and Lemma 3.7, it follows that the positive linear functional $\frac{\mathcal{R}_{f}(x)}{c} h$ dominates $\mathcal{R}_{f}$, and thus it also dominates $f$.

\section{Continuity of the Riesz-Kantorovich Functionals}

Recall again that $L$ is assumed to be equipped with a Hausdorff locally convex topology $\tau$ for which $L_{+}$is $\tau$-closed and every order interval is $\tau$-bounded. In this section we shall also assume either that

a. $L_{+}$has a $\tau$-interior point, or else that

b. $L$ is a complete metrizable topological vector space.

We have highlighted in the previous section the importance of the upper semicontinuity of the Riesz-Kantorovich functional. In this section, we shall investigate the continuity properties of functions in $\mathcal{H}_{s}^{+}$and state some basic consequences of the embedding theorem.

We start with a generalization of a well known result from the theory of Banach lattices.

Lemma 7.1. Every $\tau$-convergent sequence in $L$ has a relatively uniformly convergent subsequence. That is, if $x_{n} \stackrel{\tau}{\rightarrow} x$, then there exist a subsequence $\left\{u_{n}\right\}$ of $\left\{x_{n}\right\}$ and some $u \in L_{+}$satisfying

$$
-\frac{1}{n} u \leq u_{n}-x \leq \frac{1}{n} u
$$

for each $n$. In particular, every $\tau$-convergent sequence of $L$ has an order bounded subsequence.

Proof. Assume $x_{n} \stackrel{\tau}{\rightarrow} 0$ in $L$. Suppose first that $e$ is an interior point of $L_{+}$. In this case the conclusion follows from the fact that for each $n$ the interval $\left[-\frac{1}{n} e, \frac{1}{n} e\right]$ is a neighborhood of zero.

Next, suppose that $L$ is a Fréchet space. Choose a countable neighborhood base $\left\{U_{n}\right\}$ of zero consisting of $\tau$-closed circled sets satisfying $U_{n+1}+U_{n+1} \subseteq U_{n}$ for each $n$. Let

$$
V_{n}=U_{n} \cap L_{+}-U_{n} \cap L_{+} .
$$

By Lemma 2.1, the sets $\left\{V_{n}\right\}$ form a $\tau$-neighborhood base at zero. So, there exists a subsequence $\left\{u_{n}\right\}$ of $\left\{x_{n}\right\}$ such that $u_{n} \in \frac{1}{n} V_{n}$ (or $n u_{n} \in V_{n}$ ) holds for each $n$. For each $n$ pick $y_{n}, z_{n} \in U_{n} \cap L_{+}$such that $n u_{n}=y_{n}-z_{n}$.

Now let $w_{n}=\sum_{i=1}^{n} y_{i} \in L_{+}$and $v_{n}=\sum_{i=1}^{n} z_{i} \in L_{+}$. Clearly,

$$
w_{n+p}-w_{n} \in U_{n+1}+U_{n+2}+\cdots+U_{n+p} \subseteq U_{n}
$$


for each $n$. This implies that $\left\{w_{n}\right\}$ is a $\tau$-Cauchy sequence and hence $\left\{w_{n}\right\}$ converges to some $w$. Since $L_{+}$is closed, $w \in L_{+}$. Letting $p \rightarrow \infty$ in $w_{n+p}-w_{n} \in L_{+}$, we see that $w-w_{n} \in L_{+}$, and so $w \geq w_{n} \geq y_{n}$ for each $n$. Similarly, there exists some $v \in L_{+}$such that $v_{n} \stackrel{\tau}{\rightarrow} v$ and $z_{n} \leq v$ for each $n$. If we let $u=w+v \in L_{+}$, then we have

$$
-u \leq-v \leq-z_{n} \leq y_{n}-z_{n}=n u_{n} \leq y_{n} \leq w \leq u,
$$

and so $\frac{1}{n} u \leq u_{n} \leq \frac{1}{n} u$ holds for each $n$, as desired.

Our next result deals with the continuity at zero of the functions in $\mathcal{H}_{s}^{+}$. It is well known that for a Fréchet lattice (i.e., a locally convex-solid Riesz space with a metrizable and complete topology) the identity $L^{\prime}=L^{r}=L^{\sim}$ holds true; see [7. Theorem 8.11, p. 306].10 The next result informs us that we can do without the lattice property.

Theorem 7.2. Each function in $\mathcal{H}_{s}^{+}$is continuous at zero. In particular, we have $L^{\prime}=L^{r}=L^{\sim}$.

Proof. Fix a function $f \in \mathcal{H}_{s}^{+}$, and assume by way of contradiction that $f$ is not $\tau$-continuous at zero. This means that there exist a sequence $\left\{x_{n}\right\}$ of $L_{+}$and some $\epsilon>0$ satisfying $x_{n} \stackrel{\tau}{\rightarrow} 0$ and $f\left(x_{n}\right) \geq \epsilon>0$ for each $n$. By Lemma 7.1, we can suppose (by passing to a subsequence if necessary) that $0 \leq x_{n} \leq \frac{1}{n} u$ holds for each $n$ and some $u \in L_{+}$. But then, the monotonicity and homogeneity of $f$ imply $\frac{1}{n} f(u)=f\left(\frac{1}{n} u\right) \geq f\left(x_{n}\right) \geq \epsilon>0$ for every $n$, which is impossible. This contradiction establishes that $f$ is continuous at zero.

The preceding conclusion implies that the Riesz-Kantorovich functional of any $m$-tuple of functionals in $L^{\sim}$ is continuous at zero. So, by Theorem 6.5 we have $L^{r}=L^{\sim}$. Moreover, the above conclusion also shows that $L^{r} \subseteq L^{\prime} \subseteq L^{\sim}$. Therefore, $L^{\prime}=L^{r}=L^{\sim}$ holds true, as desired.

Corollary 7.3. The dual cone $L_{+}^{\prime}$ generates the vector space $L^{\prime}$, and the order intervals of $\left(L^{\prime}\right)^{*}$ are all $\sigma\left(\left(L^{\prime}\right)^{*}, L^{\prime}\right)$-compact.

Proof. Theorem 7.2 implies $L^{r}=L^{\prime}$. So, the cone $L_{+}^{\prime}$ is generating in $L^{\prime}$. Therefore, the vector space $\left(L^{\prime}\right)^{*}$ equipped with the topology $\sigma\left(\left(L^{\prime}\right)^{*}, L^{\prime}\right)$ is an ordered topological vector space such that its order intervals are $\sigma\left(\left(L^{\prime}\right)^{*}, L^{\prime}\right)$-closed and $\sigma\left(\left(L^{\prime}\right)^{*}, L^{\prime}\right)$-bounded-and hence they are also $\sigma\left(\left(L^{\prime}\right)^{*}, L^{\prime}\right)$-compact.

Sufficient conditions for the upper semicontinuity of the Riesz-Kantorovich functionals are presented in the next result.

Lemma 7.4. For an m-tuple $f=\left(f_{1}, f_{2}, \ldots, f_{m}\right)$ of order bounded linear functionals on $L$ we have the following.

1. The generalized Riesz-Kantorovich functional $\mathcal{R}_{f}^{*}$ is upper $\tau$-semicontinuous.

2. If the order intervals in $L$ are weakly compact, then:

(a) $\mathcal{R}_{f}=\mathcal{R}_{f}^{*}$,

(b) the Riesz-Kantorovich functional $\mathcal{R}_{f}$ is upper $\tau$-semicontinuous, and

(c) $[0, x]=\llbracket 0, x \rrbracket$ holds for each $x \in L_{+}$.

\footnotetext{
${ }^{10}$ It is also true that a given Riesz space admits at most one complete metrizable locally solid topology; see [7 Corollary 8.10, p. 306]. In particular, a Riesz space admits at most one (up to an equivalence) lattice norm under which it is a Banach lattice.
} 
Proof. Notice first that $L^{\sim}=L^{\prime}$. Therefore each $f_{i}$ is $\tau$-continuous.

(1) Take a sequence $\left\{x_{n}\right\} \subseteq L_{+}$satisfying $x_{n} \stackrel{\tau}{\rightarrow} x$ for some $x \in L_{+}$, and suppose that $\mathcal{R}_{f}^{*}\left(x_{n}\right) \geq c$ for all $n$. We need to show that $\mathcal{R}_{f}^{*}(x) \geq c$.

From Lemma 7.1 we can assume that $\left\{x_{n}\right\}$ is order bounded. That is, we can assume that there exists some $e \in L_{+}$such that $\left\{x_{n}\right\} \subseteq[0, e] \subseteq \llbracket 0, e \rrbracket$. Fix $\epsilon>0$ and for each $n$ pick some $m$-tuple $\left(z_{1}^{n}, \ldots, z_{m}^{n}\right) \in \llbracket 0, e \rrbracket^{m}$ satisfying $\sum_{i=1}^{m} z_{i}^{n} \leq x_{n}$ and

$$
\sum_{i=1}^{m} f_{i}\left(z_{i}^{n}\right)>\mathcal{R}_{f}^{*}\left(x_{n}\right)-\epsilon \geq c-\epsilon
$$

Now, according to Corollary 7.3 the order interval $\llbracket 0, e \rrbracket$ is $\sigma\left(\left(L^{\prime}\right)^{*}, L^{\prime}\right)$-compact. So, there exists a subnet $\left\{\left(z_{1}^{n_{\alpha}}, \ldots, z_{m}^{n_{\alpha}}\right)\right\}$ of the sequence $\left\{\left(z_{1}^{n}, \ldots, z_{m}^{n}\right)\right\}$ such that $z_{i}^{n_{\alpha}} \frac{\sigma\left(\left(L^{\prime}\right)^{*}, L^{\prime}\right)}{\alpha} z_{i}$ holds in $\left(L^{\prime}\right)^{*}$ for each $i$. Clearly, $z_{i} \in \llbracket 0, e \rrbracket$ for each $i$, and $\sum_{i=1}^{m} z_{i} \leq x$.

Now using $(\star)$ and the $\sigma\left(\left(L^{\prime}\right)^{*}, L^{\prime}\right)$-continuity of each $f_{i}$, we see that

$$
c-\epsilon \leq \underset{\alpha}{\limsup }\left[\sum_{i=1}^{m} f_{i}\left(z_{i}^{n_{\alpha}}\right)\right] \leq \sum_{i=1}^{m} \limsup _{\alpha} f_{i}\left(z_{i}^{n_{\alpha}}\right)=\sum_{i=1}^{m} f_{i}\left(z_{i}\right) \leq \mathcal{R}_{f}^{*}(x) .
$$

Since $\epsilon>0$ is arbitrary, we get $\mathcal{R}_{f}^{*}(x) \geq c$, as desired. This completes the proof.

(2) In this case, we can modify the preceding proof to show that $\mathcal{R}_{f}$ is also upper $\tau$-continuous. The rest follows from Lemma 6.3 and Theorem 6.4.

The next result is a simple consequence of Lemma 7.4 and Theorem 6.2

Theorem 7.5. If the supremum $g=\left[\bigvee_{i=1}^{m} f_{i}\right]^{+}$of an m-tuple of order bounded linear functionals $f=\left(f_{1}, f_{2}, \ldots, f_{m}\right)$ exists in $L^{\sim}$, then for each $x \in L_{+}$we have

$$
\left[\bigvee_{i=1}^{m} f_{i}\right]^{+}(x)=\sup \left\{\sum_{i=1}^{m} f_{i}\left(y_{i}\right): y_{i} \in \llbracket 0, x \rrbracket \text { for each } i \text { and } \sum_{i=1}^{m} y_{i} \leq x\right\} \text {. }
$$

If, in addition, the order intervals in $L$ are weakly compact, then

$$
\left[\bigvee_{i=1}^{m} f_{i}\right]^{+}(x)=\sup \left\{\sum_{i=1}^{m} f_{i}\left(y_{i}\right): y_{i} \in[0, x] \text { for each } i \text { and } \sum_{i=1}^{m} y_{i} \leq x\right\} \text {. }
$$

for each $x \in L_{+}$.

We close this section with a result that has many implications in economic theory.

Theorem 7.6. If $f=\left(f_{1}, f_{2}, \ldots, f_{m}\right)$ is an m-tuple of order bounded linear functionals, then for each $x \in L_{+}$we have

$$
\inf \left\{g(x): g \in L^{\sim} \text { and } g \geq f_{i} \text { for each } 1 \leq i \leq m\right\}=\mathcal{R}_{f}^{*}(x) .
$$

If, in addition, the order intervals in $L$ are weakly compact, then

$$
\inf \left\{g(x): g \in L^{\sim} \text { and } g \geq f_{i} \text { for each } 1 \leq i \leq m\right\}=\mathcal{R}_{f}(x) \text {. }
$$

Proof. By Lemma 7.4 we know that $\mathcal{R}_{f}^{*}$ is upper $\tau$-semicontinuous. Therefore, by Lemmas 5.2 and 7.2 we have

$$
\mathcal{R}_{f}^{*}(x)=\inf \left\{g(x): g \in L^{\sim} \text { and } g \geq \mathcal{R}_{f}^{*}\right\},
$$

for each $x \in L_{+}$. Furthermore, Lemma 6.1 guarantees that some $g \in L^{\sim}$ satisfies $g \geq \mathcal{R}_{f}^{*}$ if and only if $g \geq f_{i}$ for each $i$. This concludes the first part of the theorem. 
Now if the order intervals in $L$ are weakly compact, then by Lemma 7.4 (2) we have $\mathcal{R}_{f}=\mathcal{R}_{f}^{*}$, and the proof is finished.

\section{EXAMPLES}

Recall that the half-ray generated by a vector $x$ is the set $\mathcal{L}_{x}=\{\alpha x: \alpha \geq 0\}$. A vector $x>0$ is called extremal for the cone $L_{+}$if $[0, x]=\{\alpha x: 0 \leq \alpha \leq 1\}$, i.e., whenever the order interval $[0, x]$ coincides with the line segment joining 0 and $x$. A half-ray generated by an extremal vector is called an extremal ray of the cone $L_{+}$. Clearly, if $x$ and $y$ are extremal vectors, then either $x$ and $y$ are linearly independent, or else $\mathcal{L}_{x}=\mathcal{L}_{y}$.

Lemma 8.1. In an ordered vector space any three extremal vectors that generate three distinct extremal rays are linearly independent.

Proof. Let $x_{1}, x_{2}$, and $x_{3}$ be three extremal vectors in the cone of an ordered vector space that generate three distinct extremal rays. Assume by way of contradiction that the vectors $x_{1}, x_{2}, x_{3}$ are linearly dependent. Relabeling, we can suppose that there exist scalars $\lambda$ and $\mu$ such that $x_{1}=\lambda x_{2}+\mu x_{3}$.

If $\lambda=0$, then $x_{1}=\mu x_{3}$ and so $\mu \neq 0$. This implies $\mu>0$ and $\mathcal{L}_{x_{1}}=\mathcal{L}_{x_{3}}$, a contradiction. Hence, $\lambda \neq 0$ and $\mu \neq 0$. Obviously, the case $\lambda<0$ and $\mu<0$ cannot happen. If $\lambda>0$ and $\mu>0$, then from $0<\lambda x_{2} \leq x_{1}$, it follows that $\lambda x_{2}=\alpha x_{1}$ for some $\alpha>0$. This implies $\mathcal{L}_{x_{2}}=\mathcal{L}_{x_{1}}$, which is a contradiction. Finally, we consider the case $\lambda>0$ and $\mu<0$. In this case, we have $x_{1}<\lambda x_{2}$, and hence for some $\beta>0$ we must have $x_{1}=\beta \lambda x_{2}$. This implies $\mathcal{L}_{x_{1}}=\mathcal{L}_{x_{2}}$, which is also a contradiction. Hence, the vectors $x_{1}, x_{2}, x_{3}$ must be linearly independent.

The conclusion of the preceding result is not true for four or more extremal vectors. That is, we have the following.

- If $\mathcal{L}_{x_{1}}, \mathcal{L}_{x_{2}}, \ldots, \mathcal{L}_{x_{k}}$ are distinct extremal rays of a closed cone in an ordered vector space $L$ with $\operatorname{dim} L \geq k \geq 4$, then the vectors $x_{1}, x_{2}, \ldots, x_{k}$ need not be linearly independent.

For an example, consider the closed (convex) cone $L_{+}$in $\mathbb{R}^{4}$ generated by the four vectors

$$
x_{1}=(1,0,0,0), x_{2}=(0,1,0,0), x_{3}=(0,0,1,0), x_{4}=(1,-1,1,0) .
$$

That is,

$$
\begin{aligned}
L_{+} & =\left\{\sum_{i=1}^{4} \lambda_{i} x_{i}: \quad \lambda_{i} \geq 0 \text { for each } i\right\} \\
& =\left\{\left(\lambda_{1}+\lambda_{4}, \lambda_{2}-\lambda_{4}, \lambda_{3}+\lambda_{4}, 0\right): \lambda_{i} \geq 0 \text { for each } i\right\} .
\end{aligned}
$$

It is a routine matter to verify that $L_{+}$is indeed a cone and that the four half-rays $\mathcal{L}_{x_{1}}, \mathcal{L}_{x_{2}}, \mathcal{L}_{x_{3}}$, and $\mathcal{L}_{x_{4}}$ are the only distinct extremal rays of the cone $L_{+}$. However, notice that we have $(1,-1,1,0)=(1,0,0,0)-(0,1,0,0)+(0,0,1,0)$.

Definition 8.2. A base for the cone $L_{+}$is a non-empty convex subset of $L_{+} \backslash\{0\}$ such that for every $x>0$ there exist a scalar $\lambda>0$ and some $b \in B$, both uniquely determined, such that $x=\lambda b$.

Given a base $B$ for $L_{+}$, it is easy to see that a vector $x \in B$ is an extreme point of $B$ if and only if $x$ an extremal vector of the cone $L_{+}$. Regarding the existence 
of extreme rays, we have the following result of V. Klee [21, 22]. (For a proof see [19, Theorem 3.12.8, p. 144 and Corollary 3.12.9, p. 145].)

Lemma 8.3 (Klee). If $L$ is finite dimensional and $L_{+}$is closed, then $L_{+}$has a compact base. In particular, $L_{+}$coincides with the closed convex hull of its extremal vectors.

Two vectors $x, y \in L$ are said to be non-comparable if $x \not \leq y$ and $y \not \leq x$. The next theorem describes an interesting lattice property of non-comparable vectors in $\mathbb{R}^{3}$.

Theorem 8.4. If $L$ is three dimensional and $L_{+}$is a closed generating cone, then there are two non-comparable points $x, y \in L$ for which $x \vee y$ exists in $L$ if and only if $L_{+}$has at most four extremal rays.

Proof. It is well known that in a Hausdorff finite dimensional ordered topological vector space with a closed generating cone the order intervals are compact - for a proof see, for instance, $[16, \S 5.4]$. This implies that in our case we have $L^{\prime}=L^{\sim}$.

It is also well known that the positive cone $L_{+}$has at most four extremal rays if and only if $L_{+}^{\prime}$ also has at most four extremal rays, and that $L_{+}=L_{+}^{\prime \prime}$. Therefore, in order to prove the theorem we need only to show that there are two order noncomparable functionals $f, g \in L^{\prime}$ for which $f \vee g$ exists in $L^{\prime}$ if and only if $L_{+}$has at most four extremal rays.

To this end, fix a compact base $\mathcal{B}$ for $L_{+}$and let $\mathcal{E}$ be the set of all extreme points of $\mathcal{B}$. Therefore, aside from scalar multiples, the extremal rays of $L_{+}$can be identified with the vectors of $\mathcal{E}$. Since $L$ is three dimensional and $L_{+}$is generating, it follows from Lemma 8.3 that $|\mathcal{E}| \geq 3$, where as usual $|S|$ denotes the cardinality of the set $S$.

Now assume that there is a functional $f \notin-L_{+}^{\prime}$ and $f \notin L_{+}^{\prime}$ for which $f^{+}$exists. By Theorem 7.5 we know that $f^{+}$satisfies the Riesz-Kantorovich formula. That is, for each $x \in L_{+}$we have

$$
f^{+}(x)=\sup \{f(y): 0 \leq y \leq x\} .
$$

We want to show that $|\mathcal{E}| \leq 4$. Let

$$
\mathcal{E}_{1}=\{x \in \mathcal{E}: f(x) \geq 0\} \quad \text { and } \quad \mathcal{E}_{2}=\{x \in \mathcal{E}: f(x)<0\} .
$$

Since $f^{+}$satisfies the Riesz-Kantorovich formula, it must be the case that $f^{+}(x)=$ $f(x)$ for all $x \in \mathcal{E}_{1}$ and $f^{+}(x)=0$ for all $x \in \mathcal{E}_{2}$. Now if $\left|\mathcal{E}_{1}\right| \geq 3$, then $f^{+}=f$, since by Lemma 8.1 the set $\mathcal{E}_{1}$ spans $L$. This implies $f \geq 0$ or $f \in L_{+}^{\prime}$, which is a contradiction. Therefore, $\left|\mathcal{E}_{1}\right| \leq 2$. Similarly, if $\left|\mathcal{E}_{2}\right| \geq 3$, then $f^{+}=0$ and $f \leq 0$ or $f \in-L_{+}^{\prime}$, which is also a contradiction. Consequently, $\left|\mathcal{E}_{2}\right| \leq 2$ and hence $|\mathcal{E}| \leq 4$. We can also assume that $\mathcal{E}$ is a subset of a plane. Indeed, if we choose some interior vector $p \in L_{+}^{\prime}$, then we have $p \cdot x>0$ for each $x>0$, and so if for each $x \in \mathcal{E}$ we choose some $\lambda_{x}>0$ such that $\lambda_{x} p \cdot x=1$, then the convex hull of the set $\mathcal{E}^{\prime}=\left\{\lambda_{x} x: \quad x \in \mathcal{E}\right\}$ is a planar base of $L_{+}$; in fact the convex hull of $\mathcal{E}^{\prime}$ is either a triangle or a quadrilateral.

We shall now prove the converse. Thus, we assume that $|\mathcal{E}| \leq 4$. If $|\mathcal{E}|=3$, then $L$ is a vector lattice and the conclusion is trivially true. So, we can assume that $|\mathcal{E}|=4$. That is, let $\mathcal{E}=\left\{x_{1}, x_{2}, x_{3}, x_{4}\right\}$. Therefore,

$$
L_{+}=\left\{\sum_{i=1}^{4} \lambda_{i} x_{i}: \quad \lambda_{i} \geq 0 \text { for each } i=1,2,3,4\right\} .
$$


By relabeling the $x_{i}$ appropriately, it is not difficult to see that there exist two linear functionals $g$ and $h$ such that:

$$
g\left(x_{1}\right)=g\left(x_{2}\right)=0, g\left(x_{3}\right)>0, g\left(x_{4}\right)>0
$$

and

$$
h\left(x_{3}\right)=h\left(x_{4}\right)=0, h\left(x_{1}\right)<0, h\left(x_{2}\right)<0 .
$$

Clearly, $g>0$ and $h<0$. Put $f=g+h$ and notice that $f \not \leq 0$ and $f \geq 0$. Moreover, an easy argument shows that

$$
\begin{aligned}
\mathcal{R}_{f}\left(x_{1}\right) & =g\left(x_{1}\right)=0, & \mathcal{R}_{f}\left(x_{2}\right)=g\left(x_{2}\right)=0, \\
\mathcal{R}_{f}\left(x_{3}\right)=f\left(x_{3}\right) & =g\left(x_{3}\right)>0, & \mathcal{R}_{f}\left(x_{4}\right)=f\left(x_{4}\right)=g\left(x_{4}\right)>0 .
\end{aligned}
$$

So, if $z=\sum_{i=1}^{4} \lambda_{i} x_{i} \in L_{+}$, then $g(z)=\sum_{i=1}^{4} \lambda_{i} g\left(x_{i}\right) \geq \sum_{i=1}^{4} \lambda_{i} f\left(x_{i}\right)=f(z)$, and thus $g \geq f$. But then, the super-additivity and homogeneity of $\mathcal{R}_{f}$ for each $z=\sum_{i=1}^{4} \lambda_{i} x_{i} \in L_{+}$yields

$$
g(z) \geq \mathcal{R}_{f}(z) \geq \sum_{i=1}^{4} \lambda_{i} \mathcal{R}_{f}\left(x_{i}\right)=\sum_{i=1}^{4} \lambda_{i} g\left(x_{i}\right)=g(z) .
$$

Consequently, $g(z)=\mathcal{R}_{f}(z)$ for every $z \in L_{+}$, and this shows that $g=f^{+}$.

The next example illustrates Theorem 8.4.

Example 8.5. Consider the following four positive vectors in $L=\mathbb{R}^{3}$ :

$$
x_{1}=(0,1,0), x_{2}=(1,1,0), x_{3}=(0,1,1), x_{4}=(1,1,1) .
$$

Let $L_{+}$be the (convex) cone with vertex 0 generated by these points. That is,

$$
L_{+}=\left\{\sum_{i=1}^{4} \lambda_{i} x_{i}: \lambda_{i} \geq 0 \text { for each } i=1,2,3,4\right\} .
$$

This cone (which is a closed subcone of the standard cone $L_{+}=\mathbb{R}_{+}^{3}$ ) is not a lattice cone, but it is generating.

Now consider the vector $f=(2,-1,0)$ as a linear functional on $\mathbb{R}^{3}$. From

$$
f \cdot x_{1}=-1<0 \text { and } f \cdot x_{2}=1>0,
$$

it follows that $f$ and 0 are non-comparable linear functionals. Next, we claim that the linear functional $g=(1,0,0)$ is the least upper bound of $f$ and 0 , i.e., $g=f^{+}$ holds in $L^{\prime}=\mathbb{R}^{3}$ with respect to the ordering induced by the dual cone of $L_{+}^{\prime}$.

To see this, fix an arbitrary point

$$
z=\sum_{i=1}^{4} \lambda_{i} x_{i}=\left(\lambda_{2}+\lambda_{4}, \lambda_{1}+\lambda_{2}+\lambda_{3}+\lambda_{4}, \lambda_{3}+\lambda_{4}\right) \in L_{+} .
$$

From $g-f=(-1,1,0)$, we see that

$$
(g-f) \cdot z=(-1,1,0) \cdot\left(\lambda_{2}+\lambda_{4}, \lambda_{1}+\lambda_{2}+\lambda_{3}+\lambda_{4}, \lambda_{3}+\lambda_{4}\right)=\lambda_{1}+\lambda_{3} \geq 0 .
$$

This shows that $g \geq f$ holds in $L^{\prime}$. Moreover, $g \geq 0$ should be obvious.

To see that $g$ is the least upper bound of $f$ and 0 , notice that $\left[0, x_{i}\right]=\left\{\alpha x_{i}: 0 \leq\right.$ $\alpha \leq 1\}$ holds for $i=1,2,3,4$. This implies

$$
\begin{aligned}
& \mathcal{R}_{f}\left(x_{1}\right)=g \cdot x_{1}=0, \mathcal{R}_{f}\left(x_{2}\right)=g \cdot x_{2}=1, \\
& \mathcal{R}_{f}\left(x_{3}\right)=g \cdot x_{3}=0, \mathcal{R}_{f}\left(x_{4}\right)=g \cdot x_{4}=1 \text {. }
\end{aligned}
$$


So, if $z=\sum_{i=1}^{4} \lambda_{i} x_{i} \in L_{+}$, then $g \geq f$, and the super-additivity and homogeneity of $\mathcal{R}_{f}$ imply

$$
g \cdot z \geq \mathcal{R}_{f}(z) \geq \sum_{i=1}^{4} \lambda_{i} \mathcal{R}_{f}\left(x_{i}\right)=\sum_{i=1}^{4} \lambda_{i} g \cdot x_{i}=g \cdot z .
$$

Consequently, $g \cdot z=\mathcal{R}_{f}(z)$ for every $z \in L_{+}$, and so $g=f^{+}$holds in $L^{\prime}$.

There is another (geometrical) way of proving that $g=f^{+}$. Notice that the dual cone $L_{+}^{\prime}$ of $L_{+}$is the cone in $\mathbb{R}^{3}$ having the four extremal rays generated by the vectors

$$
(1,0,0),(-1,1,0),(0,0,1),(0,1,-1) .
$$

It is not difficult to see that $\left[(2,-1,0)+L_{+}^{\prime}\right] \cap L_{+}^{\prime}=(1,0,0)+L_{+}^{\prime}$. This implies $g=f^{+}$.

Theorem 8.4 is not true for spaces of dimension greater than three.

Example 8.6. Let $L_{+}$be an "ice-cream" cone in $\mathbb{R}^{3}$. For instance, let

$$
L_{+}=\left\{(x, y, z) \in \mathbb{R}^{3}: z \geq \sqrt{x^{2}+y^{2}}\right\} .
$$

We consider $\mathbb{R}^{4}$ partially ordered by the cone $L_{+} \times \mathbb{R}_{+}$. Notice that for any extremal ray $\mathcal{L}$ of $L_{+}$, the set $\mathcal{L} \times\{0\}$ is also an extremal ray of $L_{+} \times \mathbb{R}_{+}$. This implies that the cone $L_{+} \times \mathbb{R}_{+}$has infinitely many extremal rays.

Now let $h$ be a non-zero $L_{+}$-negative functional on $\mathbb{R}^{3}$ and put $f=(h, 1)$. It is easy to see that $f \not \leq 0$ and $f \geq 0$. However, notice that $f^{+}=(0,1)$.

\section{REFERENCES}

1. Y. A. Abramovich, Injective envelopes of normed lattices, Dokl. Akad. Nauk USSR 197 (1971), 743-745. MR 44:7257

2. Y. A. Abramovich, When each continuous operator is regular, Functional Analysis, Optimization, and Mathematical Economics, A memorial volume for L. V. Kantorovich, Oxford Univ. Press 1990, pp. 133-140. MR 91m:47051

3. Y. A. Abramovich and V. A. Geiler, On a question of Fremlin concerning order bounded and regular operators, Colloq. Math. 46 (1982), 15-17. MR 84b:46008

4. Y. A. Abramovich and A. W. Wickstead, Regular operators from and into a small Riesz space, Indag. Math. N.S. 2 (1991), 257-274. MR 93i:47052

5. Y. A. Abramovich and A. W. Wickstead, The regularity of order bounded operators into $C(K)$, II, Quart. J. Math. Oxford Ser. (2) 44 (1993), 257-270. MR 94h:47067

6. Y. A. Abramovich and A. W. Wickstead, When each continuous operator is regular, II, Indag. Math. 8 (1997), 281-294. MR 97c:47046

7. C. D. Aliprantis and K. C. Border, Infinite Dimensional Analysis: A Hitchhikers Guide, $2^{\text {nd }}$ Edition, Springer-Verlag, New York and Berlin, 1999. MR 2000k:16001

8. C. D. Aliprantis, D. J. Brown, and O. Burkinshaw, Edgeworth equilibria, Econometrica 55 (1987), 1109-1137. MR 89c:90025

9. C. D. Aliprantis, D. J. Brown, and O. Burkinshaw, Existence and Optimality of Competitive Equilibria, Springer-Verlag, Heidelberg and New York, 1990. MR 92i:90023

10. C. D. Aliprantis and O. Burkinshaw, Locally Solid Riesz Spaces, Academic Press, New York and London, 1978. MR 58:12271

11. C. D. Aliprantis and O. Burkinshaw, Positive Operators, Academic Press, New York and London, 1985. MR 87h:47086

12. C. D. Aliprantis, M. Florenzano, and R. Tourky, Economic analysis in ordered vector spaces, in preparation.

13. C. D. Aliprantis, R. Tourky, and N. C. Yannelis, The Riesz-Kantorovich formula and general equilibrium theory, J. Math. Econom. 34 (2000), 55-76. MR 2001i:91088 
14. C. D. Aliprantis, R. Tourky, and N. C. Yannelis, A theory of value with non-linear prices: equilibrium analysis beyond vector lattices, J. Econ. Theory 100 (2001), 22-72. CMP 2002:01

15. T. Andô, On fundamental properties of a Banach space with a cone, Pacific J. Math. 12 (1962), 1163-1169. MR 27:568

16. G. Debreu, Theory of Value: An Axiomatic Analysis of Economic Equilibrium, Cowles Foundation Monograph, Yale University Press, New Haven, 1959. MR 22:1447

17. G. Debreu, New concepts and techniques for equilibrium analysis, Int. Econ. Rev. 3 (1962), $257-273$.

18. T. Husain, The Open Mapping and Closed Graph Theorems in Topological Vector Spaces, Oxford Mathematical Monographs, Oxford University Press, London, 1965. MR 31:2589

19. G. J. O. Jameson, Ordered Linear Spaces, Springer-Verlag Lecture Notes in Mathematics, 141, Heidelberg and New York, 1970. MR 55:10996

20. L. V. Kantorovich, Concerning the general theory of operations in partially ordered spaces, DAN SSSR 1 (1936), 271-274 (in Russian).

21. V. L. Klee, Jr., Extremal structure of convex sets, Arch. Math. 8 (1957), 234-240. MR 19:1065a

22. V. L. Klee, Jr., Extremal structure of convex sets, II, Math. Z. 69 (1958), 90-104. MR 19:1065b

23. W. A. J. Luxemburg and A. C. Zaanen, Riesz Spaces I, North-Holland, Amsterdam, 1971. MR 58:23483

24. A. Mas-Colell, The price equilibrium existence problem in topological vector lattices, Econometrica 54 (1986), 1039-1053. MR 87m:90083

25. A. L. Peressini, Ordered Topological Vector Spaces, Harper and Row, New York and London, 1967. MR 37:3315

26. I. A. Polyrakis, Lattice-subspaces of $C[0,1]$ and positive bases, J. Math. Anal. Appl. 184 (1994), 1-18. MR 95g:46040

27. I. A. Polyrakis, Finite-dimensional lattice-subspaces of $C(\Omega)$ and curves of $\mathbb{R}^{n}$, Trans. Amer. Math. Soc. 348 (1996), 2793-2810. MR 96k:46031

28. F. Riesz, Sur quelques notions fondamentals dans la theorie générale des opérations linéaires, Ann. of Math. 41 (1940), 174-206. MR 1:147d

29. H. H. Schaefer, Topological Vector Spaces, Springer-Verlag, New York, Heidelberg, Berlin 1970. MR 33:1689 (first ed.)

30. A. C. M. van Rooij, On the space of all regular operators between two Riesz spaces, Indag. Math. 47 (1985), 95-98. MR 86k:46011

31. B. Z. Vulikh, Introduction to the Theory of Partially Ordered Spaces, Wolters-Noordhoff, Groningen, 1967. MR 37:121

Department of Economics and Department of Mathematics, Purdue University, West LAFAYETTE, InDiana 47907-1310

E-mail address: aliprantis@mgmt.purdue.edu

Department of Economics, University of Melbourne, Parkville, Victoria 3052, AusTRALIA

E-mail address: rtourky@unimelb.edu.au 